\title{
TWISTED SUMS OF SEQUENCE SPACES AND THE THREE SPACE PROBLEM ${ }^{1}$
}

BY

\author{
N. J. KALTON AND N. T. PECK
}

\begin{abstract}
In this paper we study the following problem: given a complete locally bounded sequence space $Y$, construct a locally bounded space $Z$ with a subspace $X$ such that both $X$ and $Z / X$ are isomorphic to $Y$, and such that $X$ is uncomplemented in $Z$. We give a method for constructing $Z$ under quite general conditions on $Y$, and we investigate some of the properties of $Z$.

In particular, when $Y$ is $l_{p}(1<p<\infty)$, we identify the dual space of $Z$, we study the structure of basic sequences in $Z$, and we study the endomorphisms of $Z$ and the projections of $Z$ on infinite-dimensional subspaces.
\end{abstract}

1. Introduction. Let $X$ and $Y$ be complete metric linear spaces. A twisted sum of $X$ and $Y$ is a space $Z$ which has a subspace $X^{1}$ isomorphic to $X$, with $Z / X^{1}$ isomorphic to $Y$. The twisted sum $Z$ is trivial if $X^{1}$ is complemented in $Z$; otherwise, $Z$ is nontrivial.

In this paper we give a general method of constructing nontrivial twisted sums of sequence spaces, based on a modification of a construction first described by Ribe. A special case of one of our main results is that for $0<p<\infty$ there is a nontrivial twisted sum of $l_{p}$ with itself. For $0<p<1$, this solves a problem raised in [4]. For $1<p$ the twisted sum obtained is actually a Banach space, by a result of Kalton. In particular, we have an alternate solution to the 3-space problem for Hilbert spaces, which was solved by Enflo, Lindenstrauss, and Pisier.

We summarize here the organization of the paper.

$\$ \S 2$ and 3 are technical in character; in them we collect the basic facts needed for the construction. In $\$ 2$ we show that nontrivial twisted sums arise from-and give rise to-"quasi-linear" maps. $\$ 3$ details two simplifications that can be made in the construction of quasi-linear maps. One of these reduces constructing quasi-linear maps to the problem of constructing "quasi-additive" maps; the section ends by studying quasi-additive maps on the real line.

In $\S 4$ we apply the results of $\S \S 2$ and 3 to a quite general class of sequence

Received by the editors March 2, 1978 and, in revised form, September 18, 1978. AMS (MOS) subject classifications (1970). Primary 46A10, 46B99; Secondary 46A45.

Key words and phrases. Locally bounded space, Banach space, sequence space, uncomplemented subspace, three space problem, projection, strictly singular (co-singular) operator.

${ }^{1}$ This paper was written while the first author held a visiting appointment at the University of Illinois. The second author was partially supported by an NSF grant. 
spaces. The main result of the section is that under very general conditions on the sequence space $X$, there is a nontrivial twisted sum of $X$ with itself.

In $\$ 5$ we restrict our attention to a class of twisted sums of $l_{p}(1<p<\infty)$ with itself, which we denote by $l_{p}(\phi)$. We calculate the dual space of $l_{p}(\phi)$ and then study the structure of basic sequences in $l_{p}(\phi)$. Finally, in $\S 6$ we study the spaces $Z_{p}(1<p<\infty)$ which are a subclass of the spaces $l_{p}(\phi)$. In a sense, the spaces $Z_{p}$ are "extremal" solutions to the three space problem. They yield sharp estimates for the rate of growth of projections on $l_{p}^{n}$; the inclusion map and the quotient map defining the twisted sum are, respectively, strictly co-singular and strictly singular; and the endomorphisms of $Z_{p}$ obey rather strong conditions.

We close this section by collecting some definitions and results we will need. A quasi-norm on a real vector space $X$ is a function $\|\cdot\|: X \rightarrow \mathbf{R}$ satisfying

(1) $\|x\|>0$ for $x \in X, x \neq 0$,

(2) $\|t x\|=|t|\|x\|$, for $x \in X, t \in \mathbf{R}$,

(3) $\|x+y\| \leqslant C(\|x\|+\|y\|)$, for $x, y \in X$.

The constant $C$ in (3) will be called a modulus of concavity of $\|\cdot\|$, and $(X,\|\|)$ will be called a quasi-normed space.

If we take $U=\{x \in X:\|x\| \leqslant 1\}$, then the scalar multiples of $U$ form a neighborhood base at 0 for a Hausdorff vector topology on $X$. With this topology, $X$ is locally bounded, i.e. has a bounded neighborhood of 0 . Conversely, every locally bounded topology on a vector space is given by a quasi-norm.

The locally bounded space $X$ is locally $p$-convex, $0<p \leqslant 1$, if it has a bounded neighborhood $V$ of 0 such that $\alpha x+\beta y \in V$ whenever $x, y$ are in $V$ and $|\alpha|^{p}+|\beta|^{p} \leqslant 1 . X$ is a $p$-Banach space if it is $p$-convex and complete.

Let $V$ be as above in the definition of $p$-convex. Then the gauge functional $\|\cdot\|$ of $V$ is a quasi-norm with the additional property that $x \rightarrow\|x\|^{p}$ is a subadditive function of $x$.

A fundamental result of Aoki and Rolewicz [1], [9] is

THEOREM 1.1. Every locally bounded space is p-convex for some $p>0$.

It follows that, if $(X,\|\|)$ is locally bounded, there are positive numbers $p$ and $L$ such that

$$
\left\|\sum_{i=1}^{n} x_{i}\right\| \leqslant L\left(\sum_{i=1}^{n}\left\|x_{i}\right\|^{p}\right)^{1 / p}
$$

for all $n$ and $x_{1}, \ldots, x_{n} \in X$.

Throughout, $\mathbf{R}, \mathbf{Z}$, and $\mathbf{N}$ denote, respectively, the real numbers, the integers, and the positive integers. $\mathbf{R}^{\infty}$ is the space of all finitely-supported 
real sequences. An $F$-space is a complete metrizeable linear space. The linear span of a set $A$ will be denoted by $\operatorname{lin} A$. And the symbol $i_{X}$ denotes the identity map on a space $X$.

2. Twisted sums. Suppose that $X, Y$, and $Z$ are $F$-spaces. Suppose further that $Z$ contains a subspace $X^{1}$ which is isomorphic to $X$, with $Z / X^{1}$ isomorphic to $Y$. Loosely we may say that $Z$ is a "twisted sum" of $X$ and $Y$ (in that order!). In other language, a twisted sum of the $F$-spaces $X$ and $Y$ is a short exact sequence of $F$-spaces $0 \rightarrow X \stackrel{j}{\rightarrow} Z \stackrel{q}{\rightarrow} Y \rightarrow 0$. If $X, Y$, and $Z$ are quasi-normed $F$-spaces with

$$
\|j x\|=\|x\|, \quad x \in X,
$$

and

$$
\|y\|=\inf \{\|z\|: q z=y\}, \quad y \in Y,
$$

then we say that $0 \rightarrow X \stackrel{j}{\rightarrow} Z \stackrel{q}{\rightarrow} Y \rightarrow 0$ is an isometric twisted sum of $X$ and $Y$. In this case $Z$ has a subspace $j(X)$ isometric to $X$ and $Z / j(X)$ is isometric to $Y$. In this section we collect a few basic facts about twisted sums; in particular, we show that they arise from-and give rise to-"quasi-linear" maps from $Y$ into $X$.

Let $X$ and $Y$ be fixed $F$-spaces and let $Z_{1}$ and $Z_{2}$ be two twisted sums of $X$ and $Y$.

Definition 2.1. (a) The twisted sums $Z_{1}$ and $Z_{2}$ are equivalent if there exists a continuous linear operator $T: Z_{1} \rightarrow Z_{2}$ such that the following diagram commutes:

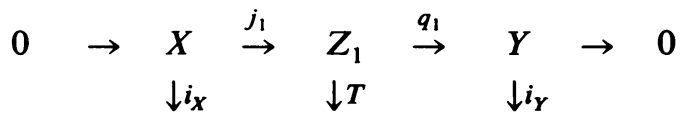

$$
\begin{aligned}
& 0 \quad \rightarrow \quad X \stackrel{j_{2}}{\rightarrow} \quad Z_{2} \stackrel{q_{2}}{\rightarrow} \quad Y \quad Y \quad \rightarrow \quad 0
\end{aligned}
$$

(b) The twisted sums $Z_{1}$ and $Z_{2}$ are projectively equivalent if there exist a continuous linear operator $T: Z_{1} \rightarrow Z_{2}$ and nonzero scalars $\alpha$ and $\beta$ so that the following diagram commutes:

$$
\begin{array}{lllllllll}
0 & \rightarrow & X & \stackrel{j_{1}}{\rightarrow} & Z_{1} & \stackrel{q_{1}}{\rightarrow} & Y & \rightarrow & 0 \\
& & \downarrow \beta i_{X} & & \downarrow T & & \downarrow \alpha i_{Y} & & \\
0 & \rightarrow & X & \stackrel{j_{2}}{\rightarrow} & Z_{2} & \stackrel{q_{2}}{\rightarrow} & Y & \rightarrow & 0
\end{array}
$$

In both (a) and (b), $T$ is necessarily one-to-one and onto, and by the open mapping theorem, $T$ is an isomorphicm.

From now on we shall restrict ourselves to locally bounded $F$-spaces. A result of Roelcke (see also Kalton [4, 1.1]) asserts that the twisted sum of locally bounded $F$-spaces is a locally bounded $F$-space. 
Proposition 2.2. Suppose $X$ and $Y$ are $p$-Banach spaces and that $Z$ is a $p$-convex twisted sum of $X$ and $Y$. Then $Z$ is projectively equivalent (and hence isomorphic) to an isometric twisted sum of $X$ and $Y$.

Proof. We may suppose that $Z$ is quasi-normed by a $p$-subadditive quasi-norm. Suppose $j: X \rightarrow Z$ and $q: Z \rightarrow Y$ are the maps inducing the twisted sum.

From the open mapping theorem there exist constants $\alpha, \beta>0$ such that

$$
\beta\|j x\| \geqslant\|x\|, \quad x \in X,
$$

and

$$
\alpha\|q z\|>\inf _{x \in X}\|z-j x\|, \quad z \in Z
$$

Now define

$$
\|z\|_{0}=\inf _{x \in X}\left(\|z-j x\|^{p}+\beta^{-p}\|x\|^{p}\right)^{1 / p}
$$

and

$$
\|z\|_{1}=\max \left(\alpha\|q z\|,\|z\|_{0}\right)
$$

Then

$$
\|z\|_{0}^{p} \leqslant\|z\|^{p}
$$

and

$$
\|z\|_{1} \leqslant(\max \{\alpha\|q\|, 1\})\|z\| .
$$

Clearly $\|\cdot\|_{1}$ is a $p$-subadditive quasi-norm on $Z$. Let $Z_{1}$ be the quasi-normed space $\left(Z,\|\|_{1}\right)$, and let $T: Z \rightarrow Z_{1}$ be the identity map. Then the following diagram commutes:

$$
\begin{array}{lllllllll}
0 & \rightarrow & X & \stackrel{j}{\rightarrow} & Z & \stackrel{q}{\rightarrow} & Y & \rightarrow & 0 \\
& & \downarrow \beta^{-i_{i_{X}}} & \downarrow T & & \downarrow \alpha i_{Y} & & \\
0 & \rightarrow & X & \stackrel{\beta j}{\rightarrow} & Z_{1} & \stackrel{\alpha q}{\rightarrow} & Y & \rightarrow & 0
\end{array} .
$$

Now,

$$
\begin{aligned}
\|\beta j x\|_{1}^{p} & =\|\beta j x\|_{0}^{p}=\inf _{x^{1} \in X}\left\{\left\|j\left(\beta x-x^{1}\right)\right\|^{p}+\beta^{-p}\left\|x^{1}\right\|^{p}\right\} \\
& >\inf _{x^{1} \in X}\left\{\beta^{-p}\left\|\beta x-x^{1}\right\|^{p}+\beta^{-p}\left\|x^{1}\right\|^{p}\right\} \\
& =\|x\|^{p}=\beta^{-p}\|\beta x\|^{p}>\|\beta j x\|_{0}^{p} .
\end{aligned}
$$

Hence $\beta j$ is an isometry. Clearly $\|\alpha q z\| \leqslant\|z\|_{1}$. For $\varepsilon>0$ and $z \in Z$ we may choose $x \in X$ so that

$$
\|z-j x\| \leqslant(1+\varepsilon) \alpha\|q z\|=(1+\varepsilon)\|\alpha q z\| .
$$

Hence $\|z-j x\|_{1}<(1+\varepsilon)\|\alpha q z\|$. 
These conditions together imply that $Z_{1}$ is complete, that it is an isometric twisted sum of $X$ and $Y$, and that it is projectively equivalent to $Z$. The proof is complete.

Definition 2.3. Let $F$ be a map from $Y$ to $X$. The map $F$ is quasi-linear if it satisfies

(a) $F(t y)=t F(y)$ for $y \in Y, t \in \mathbf{R}$, and

(b) $\left\|F\left(y_{1}+y_{2}\right)-F\left(y_{1}\right)-F\left(y_{2}\right)\right\| \leqslant M\left(\left\|y_{1}\right\|+\left\|y_{2}\right\|\right)$

for $y_{1}, y_{2} \in Y$, where $M$ is a constant independent of $y_{1}$ and $y_{2}$.

Given any quasi-linear map $F: Y \rightarrow X$ we can construct a twisted sum $X \oplus_{F} Y$ as follows: $X \oplus_{F} Y$ is the vector space $X \oplus Y$ with the quasi-norm $\|(x, y)\|=\|y\|+\|x-F(y)\|$. (We are using the same symbol $\|\cdot\|$ to denote the quasi-norm on $X \oplus Y, Y$, and $X$.) It is easy to see that the maps $j x=(x, 0)$ and $q(x, y)=y$ define, respectively, an embedding of $X$ into $X \oplus_{F} Y$ and a quotient map of $X \oplus_{F} Y$ onto $Y$. It follows that $X \oplus_{F} Y$ is complete and hence a twisted sum. This construction was first described by Ribe [7]; a very similar construction is also given by Kalton [4, 4.6]. The following converse result is implicit in Proposition 3.3 of [4]:

THEOREM 2.4. If $Z$ is a twisted sum of $X$ and $Y$, then there is a quasi-linear map $F: Y \rightarrow X$ such that $Z$ is equivalent to $X \oplus_{F} Y$.

Proof. Let $C$ be a modulus of concavity for the quasi-norms on $X, Y$, and $Z$. Let $0 \rightarrow X \stackrel{j}{\rightarrow} Z \stackrel{q}{\rightarrow} Y \rightarrow 0$ define the twisted sum. Then there is a linear, possibly discontinuous, map $\theta: Y \rightarrow Z$ such that $q \theta=i_{Y}$. Since $q$ is open there are a constant $K$ and a map $\phi: Y \rightarrow Z$ such that $\|\phi(y)\|<K\|y\|$ and $q \phi(y)=y$ for all $y \in Y$. We may suppose that $\phi(t y)=t \phi(y)$ for $y \in Y$.

Now let $F(y)=j^{-1}(\phi(y)-\theta(y))$. Let $L$ be such that $\left\|j^{-1} z\right\|<L\|z\|$ for $z \in j(X)$. Then for $y_{1}, y_{2} \in Y$,

$$
\begin{aligned}
\left\|F\left(y_{1}+y_{2}\right)-F\left(y_{1}\right)-F\left(y_{2}\right)\right\| & =\left\|j^{-1}\left(\phi\left(y_{1}+y_{2}\right)-\phi\left(y_{1}\right)-\phi\left(y_{2}\right)\right)\right\| \\
& \leqslant L\left\|\phi\left(y_{1}+y_{2}\right)-\phi\left(y_{1}\right)-\phi\left(y_{2}\right)\right\| \\
& \leqslant C^{2} L\left(\left\|\phi\left(y_{1}+y_{2}\right)\right\|+\left\|\phi\left(y_{1}\right)\right\|+\left\|\phi\left(y_{2}\right)\right\|\right) \\
& \leqslant 2 C^{3} K L\left(\left\|y_{1}\right\|+\left\|y_{2}\right\|\right) .
\end{aligned}
$$

Hence $F$ is quasi-linear.

Now define $T: Z \rightarrow X \oplus_{F} Y$ by $T z=\left(j^{-1}(z-\theta q z), q z\right)$. Then

$$
\begin{aligned}
\|T z\| & =\left\|j^{-1}(z-\phi(q z)), q z\right\| \leqslant L\|z-\phi(q z)\|+\|q z\| \\
& \leqslant C L(\|z\|+\|\phi(q z)\|)+\|q z\| \leqslant(C L+K\|q\|+\|q\|)\|z\| .
\end{aligned}
$$

Hence $T$ is continuous. It is easy to see that $T$ induces an equivalence between $Z$ and $X \oplus_{F} Y$; the proof is complete.

We shall say that two quasi-linear maps $F, G: Y \rightarrow X$ are (projectively) 
equivalent if $X \oplus_{F} Y$ and $X \oplus_{G} Y$ are (projectively) equivalent twisted sums. Note that if $F \equiv 0$ on $Y$ then $F$ induces the ordinary topological direct sum $X \oplus Y$. We shall say that $F$ is trivial if it is equivalent to the identically zero map. In this case the short exact sequence $0 \rightarrow X \rightarrow X \oplus_{F} Y \rightarrow Y \rightarrow 0$ splits.

THEOREM 2.5. (a) The maps $F$ and $G$ are equivalent if and only if there exist a constant $M$ and a linear map $A: Y \rightarrow X$ such that

$$
\|F(y)-G(y)-A(y)\| \leqslant M\|y\|, \quad y \in Y ;
$$

(b) $F$ and $G$ are projectively equivalent if and only if there exist constants $M$ and $\alpha, \alpha \neq 0$, such that

$$
\|F(y)-G(\alpha y)-A(y)\| \leqslant M\|y\|, \quad y \in Y
$$

(c) $F$ is trivial if and only if it is projectively equivalent to the zero map.

Proof. (a) If $F$ and $G$ are equivalent there is a continuous linear map $T$ : $X \oplus_{F} Y \rightarrow X \oplus_{G} Y$ of the form $T(x, y)=(x+A y, y)$, where $A: Y \rightarrow X$ is linear. Then $T(F(y), y)=(F(y)+A y, y)$ and hence

$$
\|F(y)-G(y)+A y\| \leqslant\|T\|\|y\| .
$$

Conversely, if $F, G$ and $A$ satisfy the hypothesis, define $T: X \oplus_{F} Y \rightarrow$ $X \oplus_{G} Y$ by $T(x, y)=(x+A y, y)$. It is easy to check that $T$ is continuous and induces an equivalence between $X \oplus_{F} Y$ and $X \oplus_{G} Y$.

The proof of $(b)$ is similar, and (c) is obvious.

3. Simplification of the construction of twisted sums. There are two important simplifications of the construction of quasi-linear maps and hence of twisted sums. The first allows us to consider quasi-linear maps defined only on a dense subspace of $Y$; the second allows us to drop the requirement of homogeneity in the definition of quasi-linear map.

THEOREM 3.1. Suppose that $X$ and $Y$ are quasi-normed $F$-spaces and that $Y_{0}$ is a dense subspace of $Y$. Suppose $F_{0}: Y_{0} \rightarrow X$ is a quasi-linear map. Then

(i) there is a quasi-linear map $F: Y \rightarrow X$ such that $F(y)=F_{0}(y), y \in Y_{0}$;

(ii) if $F^{*}: Y \rightarrow X$ is any quasi-linear map such that $F^{*}(y)=F_{0}(y), y \in Y_{0}$, then $F^{*}$ and $F$ are equivalent.

Proof. Consider the space $X \oplus_{F_{0}} Y_{0}$ quasi-normed by

$$
\|(x, y)\|=\left\|x-F_{0}(y)\right\|+\|y\| .
$$

Let $Z$ be the completion of this space; then $Z$ is a twisted sum of $X$ and $Y$. To see this, define $j: X \rightarrow X \oplus_{F_{0}} Y_{0}$ by $j(x)=(x, 0)$ and $\hat{q}: X \oplus_{F_{0}} Y_{0} \rightarrow Y$ by $\hat{q}(x, y)=y$. Then $\hat{q}$ extends to an open mapping $q$ of $Z$ onto $Y$ whose kernel is precisely $j(X)$. For this last point, note that (as in [4, 3.3]) if $\left(x_{n}, y_{n}\right)$ is a sequence in $X \oplus_{F_{0}} Y_{0}$ converging to $z$ in $Z$ and $\hat{q}\left(x_{n}, y_{n}\right)=y_{n} \rightarrow 0$, then 
$\left(F_{0}\left(y_{n}\right), y_{n}\right) \rightarrow 0$ and hence $\left(x_{n}-F_{0}\left(y_{n}\right), 0\right) \rightarrow z$. Thus $\left(x_{n}-F_{0}\left(y_{n}\right)\right)$ converges to some $x_{0}$ in $X$ and $z=j\left(x_{0}\right)$.

Now by Theorem 2.4, $Z$ is equivalent to $X \oplus_{H} Y$ for some quasi-linear $H$, i.e. there exists $T: Z \rightarrow X \oplus_{H} Y$ such that the following diagram commutes:

$$
\begin{array}{llllcllll}
0 & \rightarrow & X & \stackrel{j}{\rightarrow} & Z & \stackrel{q}{\rightarrow} & Y & \rightarrow & 0 \\
& & & \downarrow i_{X} & & \downarrow T & & \downarrow i_{Y} & \\
0 & \rightarrow & X & \rightarrow & X \oplus_{H} Y & \rightarrow & Y & \rightarrow & 0
\end{array}
$$

Then $T \mid X \oplus_{F_{0}} Y_{0}$ has the form $T(x, y)=(x+A y, y)$ where $A: Y_{0} \rightarrow X$ is linear. From this,

$$
\left\|F_{0}(y)+A(y)-H(y)\right\| \leqslant\|T\|\|y\|, \quad y \in Y_{0} .
$$

If we define

$$
\begin{aligned}
F(y) & =H(y)-A(y), \quad y \notin Y_{0}, \\
& =F_{0}(y), \quad y \in Y_{0},
\end{aligned}
$$

then $F$ is a quasi-linear extension of $F$. Uniqueness up to equivalence is guaranteed by the fact that any extension of $F_{0}$ defines a completion of $X \oplus_{F_{0}} Y_{0}$. The proof of Theorem 3.1 is complete.

Let $Y_{0}$ be a dense subspace of $Y$.

Definition 3.2. A map $f: Y_{0} \rightarrow X$ is quasi-additive if it satisfies

(i) $\left\|f\left(y_{1}+y_{2}\right)-f\left(y_{1}\right)-f\left(y_{2}\right)\right\| \leqslant K\left(\left\|y_{1}\right\|+\left\|y_{2}\right\|\right), y_{1}, y_{2} \in Y_{0}$;

(ii) $\lim _{t \rightarrow 0} f(t y)=0, y \in Y_{0}$;

(iii) $f(-y)=-f(y), y \in Y_{0}$.

Thus we weaken the homogeneity assumption of quasi-linearity, replacing it by antisymmetry (iii) and continuity at zero along lines (ii). With $K$ the constant in (i), we say that $f$ is quasi-additive of order $K$.

The following lemma is a simple modification of Lemma 3.2 of [4]:

Lemma 3.3. Suppose $X$ and $Y$ are quasi-normed $F$-spaces. Then there exist positive constants $r$ and $L$ such that whenever $Y_{0}$ is a dense subspace of $Y$ and $f$ : $Y_{0} \rightarrow X$ is quasi-additive of order $K$,

$$
\left\|f\left(\sum_{i=1}^{n} y_{i}\right)-\sum_{i=1}^{n} f\left(y_{i}\right)\right\| \leqslant K L\left(\sum_{i=1}^{n}\left\|y_{i}\right\|^{r}\right)^{1 / r}
$$

for any $y_{1}, \ldots, y_{n} \in Y_{0}$. If $X=Y=\mathbf{R}$, then $L$ can be taken to equal 1 and $r$ can be taken to equal $\frac{1}{2}$.

LEMMA 3.4. Suppose $X$ is a quasi-normed $F$-space. Then there is a constant $B_{X}$ depending on $X$ such that whenever $f: \mathbf{R} \rightarrow X$ is quasi-additive of order $K$, then

$$
\|f(t)-t f(1)\| \leqslant B_{X} K, \quad 0 \leqslant t \leqslant 1 .
$$


Proof. By replacing $f$ by $g(t)=f(t)-t f(1)$, we see that it suffices to consider the case when $f(1)=0$. In this case,

$$
\left\|f\left(2^{-n}\right)-2 f\left(2^{-(n+1)}\right)\right\|<K 2^{-n}
$$

and hence

$$
\left\|2^{n} f\left(2^{-n}\right)-2 f\left(2^{-(n+1)}\right)\right\|<K .
$$

Now $L$ and $r$ can be chosen satisfying Lemma 3.3 with $Y=\mathbf{R}$ and also such that

$$
\left\|\sum_{i=1}^{n} x_{i}\right\| \leqslant L\left(\sum_{i=1}^{n}\left\|x_{i}\right\|^{r}\right)^{1 / r}
$$

for $x_{1}, \ldots, x_{n} \in X$ (by Theorem 1.1). We then have

$$
\left\|2^{n} f\left(2^{-n}\right)\right\|<L K n^{1 / r}, \quad n \in \mathbf{N} \text {. }
$$

Let $t$ be in $(0,1)$; we suppose that $2^{-n}<t<2^{1-n}$ for some integer $n$. Then

$$
t=2^{-n} \sum_{k=0}^{\infty} \varepsilon_{k} 2^{-k}
$$

where $\varepsilon_{0}=1$ and each $\varepsilon_{k}$ is either 0 or 1 . Let

$$
t_{m}=2^{-n} \sum_{k=0}^{m} \varepsilon_{k} 2^{-k}
$$

Then

$$
\left\|f\left(t_{m}\right)-\sum_{k=0}^{m} \varepsilon_{k} f\left(2^{-(n+k)}\right)\right\|<K L\left(\sum_{k=0}^{m} 2^{-(n+k) r}\right)^{1 / r}<K L\left(\frac{1}{1-2^{-r}}\right)^{1 / r} .
$$

From this,

$$
\begin{aligned}
\left\|f\left(t_{m}\right)\right\| & <K L^{2}\left[\left(1-2^{-r}\right)^{-1}+\sum_{k=0}^{m} \varepsilon_{k}(n+k) 2^{-(n+k) r}\right]^{1 / r} \\
& <K L^{2}\left[\left(1-2^{-r}\right)^{-1}+\sum_{k=0}^{\infty} k 2^{-k r}\right]^{1 / r}=L^{*} K, \text { say. }
\end{aligned}
$$

Finally, $\left\|f(t)-f\left(t_{m}\right)-f\left(t-t_{m}\right)\right\|<K$ and hence

$$
\begin{aligned}
\|f(t)\| & <L\left(\left\|f\left(t_{m}\right)\right\|^{r}+K^{r}\left\|f\left(t-t_{m}\right)\right\|^{r}+K^{r}\right)^{1 / r} \\
& <L K\left(\left(L^{*}\right)^{r}+1+\left\|f\left(t-t_{m}\right)\right\|^{r}\right)^{1 / r}
\end{aligned}
$$

Since $f\left(t-t_{m}\right) \rightarrow 0$, we have, on letting $m \rightarrow \infty$, that

$$
\|f(t)\| \leqslant L\left(\left(L^{*}\right)^{r}+1\right)^{1 / r} K
$$

and the result is proved. 
THEOREM 3.5. Suppose $X$ and $Y$ are quasi-normed $F$-spaces and $Y_{0}$ is a dense subspace of $Y$. Then if $f: Y_{0} \rightarrow X$ is quasi-additive, the map $F: Y_{0} \rightarrow X$ defined by

$$
\begin{aligned}
F(x) & =\|x\| f(x /\|x\|), \quad x \neq 0, \\
& =0, \quad x=0,
\end{aligned}
$$

is quasi-linear.

Proof. If $\|x\| \leqslant 1$ and $f$ is quasi-additive of order $K$, then

$$
\|F(x)-f(x)\|<B_{X} K
$$

by Lemma 3.4. Let $C$ denote a modulus of concavity for $X$. Then if $\|x\|+\|y\| \leqslant 1 / C$, we have $\|x+y\| \leqslant 1$ and so

$$
\|F(x+y)-f(x+y)\| \leqslant B_{X} K .
$$

Now,

$$
\|F(x+y)-F(x)-F(y)\| \leqslant C\left(\|f(x+y)-f(x)-f(y)\|+3 C^{2} B_{X} K\right) .
$$

Hence

$$
\|F(x+y)-F(x)-F(y)\| \leqslant C\left(K+3 C^{2} B_{X} K\right) .
$$

Thus $F$ is quasi-linear, and the proof is complete.

We now turn to the form of quasi-additive maps from $\mathbf{R}$ into $\mathbf{R}$. Let $B=B_{R}$ (as obtained in Lemma 3.4).

LEMMA 3.6. If $f: \mathbf{R} \rightarrow \mathbf{R}$ and $g: \mathbf{R} \rightarrow \mathbf{R}$ are quasi-additive of order $K$ and

$$
\left|f\left(2^{n}\right)-g\left(2^{n}\right)\right| \leqslant M \cdot 2^{n}, \quad n \in \mathbf{Z}
$$

then

$$
|f(t)-g(t)| \leqslant(M+4 B K)|t|, \quad t \in \mathbf{R} .
$$

Proof. For $t$ in $\mathbf{R}^{+}$, choose $n$ in $\mathbf{Z}$ so that $2^{n-1}<t<2^{n}$, and define $f^{(n)}(r)=f\left(2^{n} r\right), g^{(n)}(r)=g\left(2^{n} r\right), r \in \mathbf{R}$. The functions $f^{(n)}$ and $g^{(n)}$ are quasiadditive of order $2^{n} K$. Hence

$$
\begin{aligned}
& \left|f^{(n)}\left(2^{-n} t\right)-2^{-n} t f^{(n)}(1)\right|<2^{n} B K, \\
& \left|g^{(n)}\left(2^{-n} t\right)-2^{-n} t^{(n)}(1)\right| \leqslant 2^{n} B K .
\end{aligned}
$$

Then

$$
\begin{aligned}
|f(t)-g(t)| & \leqslant 2^{-n} t\left|f\left(2^{n}\right)-g\left(2^{n}\right)\right|+2^{n+1} B K \\
& \leqslant(M+4 B K) t
\end{aligned}
$$

This proves the lemma for nonnegative $t$; since $f$ and $g$ are antisymmetric, we have the result for all real $t$. 
TheOREM 3.7. (a) If $\boldsymbol{\theta}: \mathbf{R} \rightarrow \mathbf{R}$ is a Lipschitz function, then the map

$$
\begin{aligned}
f(t) & =t \theta(\log (1 /|t|)), \quad t \neq 0, \\
& =0, \quad t=0,
\end{aligned}
$$

is quasi-additive, of order $L \log 2$ where $L$ is the Lipschitz constant of $\theta$.

(b) Conversely, if $f$ is quasi-additive there exists a Lipschitz function $\theta$ : $\mathbf{R} \rightarrow \mathbf{R}$ such that

$$
\sup _{0<t<\infty}\left|\frac{f(t)}{t}-\theta\left(\log \frac{1}{|t|}\right)\right|<\infty
$$

Proof. (a) For $t_{1}, t_{2}>0$,

$$
\begin{aligned}
& \left|\frac{f\left(t_{1}+t_{2}\right)-f\left(t_{1}\right)-f\left(t_{2}\right)}{t_{1}+t_{2}}\right| \\
& =\left|\theta\left(\log \frac{1}{t_{1}+t_{2}}\right)-\frac{t_{1}}{t_{1}+t_{2}} \theta\left(\log \frac{1}{t_{1}}\right)-\frac{t_{2}}{t_{1}+t_{2}} \theta\left(\log \frac{1}{t_{2}}\right)\right| \\
& \leqslant L\left(\frac{t_{1}}{t_{1}+t_{2}} \log \frac{t_{1}+t_{2}}{t_{1}}+\frac{t_{2}}{t_{1}+t_{2}} \log \frac{t_{1}+t_{2}}{t_{2}}\right)
\end{aligned}
$$

where $L$ is the Lipschitz constant of $\theta$. Since

$$
t \log \frac{1}{t}+(1-t) \log \frac{1}{1-t} \leqslant \log 2, \quad 0<t<1,
$$

we conclude that

$$
\left|f\left(t_{1}+t_{2}\right)-f\left(t_{1}\right)-f\left(t_{2}\right)\right| \leqslant L \log 2\left(t_{1}+t_{2}\right) .
$$

It now follows easily that $f$ is quasi-additive.

(b) If $f$ is quasi-additive of order $K$, then

$$
\left|f\left(2^{n+1}\right)-2 f\left(2^{n}\right)\right| \leqslant K \cdot 2^{n+1}, \quad n \in \mathbf{Z}
$$

Hence

$$
\left|\frac{f\left(2^{n+1}\right)}{2^{n+1}}-\frac{f\left(2^{n}\right)}{2^{n}}\right|<K
$$

Now choose $\theta$ a Lipschitz function of Lipschitz constant $K$ such that $\theta(n)=2^{n} f\left(2^{-n}\right), n \in \mathbf{Z}$, and define

$$
\begin{aligned}
g(t) & =t \theta(-\log |t| / \log 2), \quad t \neq 0, \\
& =0, \quad t=0 .
\end{aligned}
$$

Then $g$ is quasi-additive of order $K$ and $f\left(2^{n}\right)=g\left(2^{n}\right), n \in \mathbf{Z}$. Thus

$$
\left|\frac{f(t)}{t}-\frac{g(t)}{t}\right| \leqslant 2 B K, \quad t \neq 0,
$$

by the preceding lemma, and the result follows. 
4. Twisted sums of sequence spaces. In this section we apply the results of the preceding two sections to the construction of quasi-linear functions on sequence spaces. We obtain that under very general conditions on the sequence space $E$, there is a short exact sequence

$$
0 \rightarrow E \rightarrow Z \rightarrow E \rightarrow 0
$$

which does not split. In particular, we give an alternate proof of the solution of the 3-space problem for Hilbert spaces.

Definition 4.1. A solid quasi-normed $F K$-space is a sequence space $E$ satisfying:

(i) if $e_{n}$ denotes the $n$th basis vector $\left(e_{n}(k)=\delta_{n k}\right)$, then the linear span $\mathbf{R}^{\infty}$ of $\left(e_{n}\right)$ is dense in $E$;

(ii) the quasi-norm $\|\cdot\|$ on $E$ satisfies

(a) $\|s x\| \leqslant\|s\|_{\infty}\|x\|, s \in l_{\infty}, x \in E$;

(b) $\left\|e_{n}\right\|=1, n \in \mathbf{N}$;

(c) $\|x\|_{\infty} \leqslant\|x\|, x \in E$.

Under these circumstances $\left(e_{n}\right)$ is an unconditional basis for $E$.

Notation. Let $\mathcal{E}$ denote the class of Lipschitz functions $\phi: \mathbf{R} \rightarrow \mathbf{R}$ such that $\phi(t)=0$ for $t \leqslant 0$.

For $\phi$ in $\mathcal{L}$, we define a quasi-additive map $f: \mathbf{R}^{\infty} \rightarrow \mathbf{R}^{\infty}$ by

$$
\begin{aligned}
f(x)[k] & =x(k) \phi(-\log |x(k)|), \quad x(k) \neq 0, \quad k \in \mathbf{N}, \\
& =0, \quad x(k)=0, \quad k \in \mathbf{N} .
\end{aligned}
$$

To see that $f$ is quasi-additive (for the quasi-norm on $E$ ), observe that if $L$ is the Lipschitz constant of $\phi$, then for $x_{1}, x_{2}$ in $E$,

$$
\left|f\left(x_{1}\right)[k]+f\left(x_{2}\right)[k]-f\left(x_{1}+x_{2}\right)[k]\right| \leqslant L \log 2\left(\left|x_{1}(k)+x_{2}(k)\right|\right) .
$$

From this,

$$
\left|f\left(x_{1}\right)+f\left(x_{2}\right)-f\left(x_{1}+x_{2}\right)\right| \leqslant L \log 2\left(\left|x_{1}\right|+\left|x_{2}\right|\right)
$$

(using the obvious lattice operations on $E$ ). Thus

$$
\begin{aligned}
\left\|f\left(x_{1}\right)+f\left(x_{2}\right)-f\left(x_{1}+x_{2}\right)\right\| & \leqslant L \log 2\left(\left\|\left|x_{1}\right|+\left|x_{2}\right|\right\|\right) \\
& \leqslant C L \log 2\left(\left\|x_{1}\right\|+\left\|x_{2}\right\|\right)
\end{aligned}
$$

where $C$ is a modulus of concavity of $\|\cdot\|$.

Next, by Theorems 3.5 and 3.1, $f$ induces a quasi-linear map $F_{0}: \mathbf{R}^{\infty} \rightarrow \mathbf{R}^{\infty}$ :

$$
\begin{aligned}
F_{0}(x) & =\|x\| f(x /\|x\|), \quad x \neq 0, \\
& =0, \quad x=0,
\end{aligned}
$$

and $F_{0}$ may be extended to a quasi-linear map $F: E \rightarrow E$. We now study the properties of the twisted sum $E \oplus_{F} E$.

Note first that there is no gain of generality in relaxing the restriction $\phi(t)=0, t \leqslant 0$; each $\phi$ may be replaced by 


$$
\begin{aligned}
\bar{\phi}(t) & =\phi(t)-\phi(0), \quad t>0, \\
& =0, \quad t \leqslant 0,
\end{aligned}
$$

and it is easily seen that $\bar{\phi}$ induces a twisted sum equivalent to $E \oplus_{F} E$. Secondly, note that although $F$ is not unique, it is unique up to equivalence.

Notation. For $\phi$ in $\mathcal{L}, E(\phi)$ denotes the (unambiguously defined) twisted sum $E \oplus_{F} E$.

THEOREM 4.2. Let $E$ be a solid quasi-normed $F K$-space such that no subsequence of $\left(e_{n}\right)$ is equivalent to the unit vector basis of $c_{0}$. Let $\phi$ and $\psi$ be in $\mathcal{L}$. Then

(a) the two twisted sums $E(\phi)$ and $E(\psi)$ are equivalent if and only if

$$
\sup _{0<t<\infty}|\phi(t)-\psi(t)|<\infty ;
$$

(b) $E(\phi)$ and $E(\psi)$ are projectively equivalent if and only if for some $a \neq 0$,

$$
\sup _{0<t<\infty}|\phi(t)-a \psi(t)|<\infty
$$

(c) $E(\phi)$ is trivial if and only if $\phi$ is bounded.

Proof. (a) Suppose $\phi$ and $\psi$ induce the quasi-linear maps $F: E \rightarrow E$ and $G$ : $E \rightarrow E$. Then by $2.5, E(\phi)$ and $E(\psi)$ are equivalent if and only if there is a linear map $A: E \rightarrow E$ such that

$$
\|F(y)-G(y)-A y\| \leqslant M\|y\|, \quad y \in E .
$$

It is immediate that if

$$
\sup _{0<t<\infty}|\phi(t)-\psi(t)|<\infty
$$

the above condition holds with $a=0$.

For the proof of the converse, suppose there is a linear map $A: E \rightarrow E$ such that

$$
\|F(y)-G(y)-A y\| \leqslant M\|y\|, \quad y \in E .
$$

Let $H$ be the quasi-linear map $F-G$. Observe that $H\left(e_{n}\right)=0$ for all $n$, and hence $\left\|A e_{n}\right\| \leqslant M, n \in \mathrm{N}$. In particular, $\left\|A e_{n}\right\|_{\infty}<M, n \in \mathrm{N}$, and so we may select increasing sequences of integers $(n(k))$ and $(m(k))$ such that $A\left(e_{n(k)}-\right.$ $\left.e_{m(k)}\right) \rightarrow 0$ coordinate-wise.

Now by a standard gliding hump argument we may select a subsequence $\left(f_{n}\right)_{n=1}^{\infty}$ of $\left(e_{n(k)}-e_{m(k)}\right)_{k=1}^{\infty}$ and an increasing sequence $\left(p_{n}\right)$ such that $p_{0}=0$ and

(1) $\left\|A f_{n}-\sum_{k=p_{n-1}+1}^{p_{n}}\left(A f_{n}(k)\right) e_{k}\right\|<2^{-n}$;

(2) $\operatorname{supp}\left\{f_{n}\right\}=\left\{q_{n}, r_{n}\right\} \subset\left\{p_{n-1}+1, \ldots, p_{n}\right\}$.

Define a linear map $\hat{A}: \operatorname{lin}\left(f_{n}\right) \rightarrow E$ by

$$
\hat{A} f_{n}=\left(A f_{n}\right)\left(q_{n}\right) e_{q_{n}}+\left(A f_{n}\right)\left(r_{n}\right) e_{r_{n}} .
$$


Then $\left\|\hat{A} f_{n}\right\| \leqslant 2 C M$, where $C$ is a modulus of concavity for $\|\cdot\|$. Hence, $\left\|\hat{A} f_{n}\right\|_{\infty}<2 C M$, and so $\left|\hat{A} f_{n}\right| \leqslant 2 C M\left|f_{n}\right|, n \in \mathbf{N}$.

For $x=\sum_{n=1}^{N} t_{n} f_{n}$ in $\operatorname{lin}\left(f_{n}\right)$, we have $\max \left|t_{n}\right|<\|x\|_{\infty}<\|x\|$. Hence

$$
\left\|A x-\sum_{n=1}^{N} t_{n} \sum_{k=p_{n-1}+1}^{p_{n}}\left(A f_{n}(k)\right) e_{k}\right\|<M_{1}\|x\|,
$$

where $M_{1}$ depends only on the modulus of concavity of $\|\cdot\|$ (This follows from (1) above and Theorem 1.1: if $r$ and $L$ are chosen as in Theorem 1.1 and $\left\|y_{i}\right\|<2^{-i}$, then

$$
\left\|\sum_{i=1}^{N} y_{i}\right\| \leqslant L\left(\sum_{i=1}^{N}\left\|y_{i}\right\|^{r}\right)^{1 / r} \leqslant L\left(1-2^{-r}\right)^{-1 / r}
$$

for any $N$.) Thus

$$
\left\|H(x)-\sum_{n=1}^{N} t_{n} \sum_{k=p_{n-1}+1}^{p_{n}}\left(A f_{n}(k)\right) e_{k}\right\| \leqslant M_{2}^{*}\|x\|
$$

and hence

$$
\left\|H(x)-\sum_{n=1}^{N} t_{n} \hat{A} f_{n}\right\|<M_{2}\|x\|,
$$

with $M_{2}^{*}$ and $M_{2}$ independent of $x$.

Now let $s_{n}=f_{1}+\cdots+f_{n}, n \in \mathbf{N}$. Then

$$
\left\|H\left(s_{n}\right)-\sum_{i=1}^{n} \hat{A} f_{i}\right\| \leqslant M_{2}\left\|s_{n}\right\| \text {. }
$$

However, $H\left(s_{n}\right)=\left(\phi\left(\log \left\|s_{n}\right\|\right)-\psi\left(\log \left\|s_{n}\right\|\right)\right) s_{n}$ and so

$$
\left\|\left|\phi\left(\log \left\|s_{n}\right\|\right)-\psi\left(\log \left\|s_{n}\right\|\right)\right|\left|s_{n}\right|-\sum_{i=1}^{n}\left|\hat{A} f_{i}\right|\right\|<M_{2}\left\|s_{n}\right\|
$$

for all $n$. Further,

$$
\sum_{i=1}^{n}\left|\hat{A} f_{i}\right| \leqslant 2 C M\left|s_{n}\right|, \quad n \in \mathbf{N}
$$

and hence if $\left|\phi\left(\log \left\|s_{n}\right\|\right)-\psi\left(\log \left\|s_{n}\right\|\right)\right|>2 C M$, then

$$
\left|\phi\left(\log \left\|s_{n}\right\|\right)-\psi\left(\log \left\|s_{n}\right\|\right)\right|-2 C M<M_{2} .
$$

Thus

$$
\left|\phi\left(\log \left\|s_{n}\right\|\right)-\psi\left(\log \left\|s_{n}\right\|\right)\right| \leqslant 2 C M+M_{2}, \quad n \in N .
$$

Note that if $\sup _{n}\left\|s_{n}\right\|<\infty$, the sequence $\left\{e_{q_{1}}, e_{r_{1}}, e_{q_{2}}, e_{r_{2}}, \ldots\right\}$ is equivalent to the usual basis of $c_{0}$. For assume $\sup _{n}\left\|s_{n}\right\|=S$ is finite; then if $x=\Sigma_{i} t_{i} e_{q_{i}}+\Sigma_{j} t_{j} e_{r_{j}}$,

$$
\max _{i, j}\left\{\left|t_{i}\right|,\left|t_{j}\right|\right\}<\|x\|<S \cdot \max _{i, j}\left\{\left|t_{i}\right|,\left|t_{j}\right|\right\}
$$


Hence from our assumption it follows that $\left\|s_{n}\right\| \rightarrow \infty$. Also, $\left\|f_{n}\right\|<2 C$ and hence

$$
\left\|s_{n}\right\| \leqslant C\left(\left\|s_{n-1}\right\|+2 C\right), \quad n \in N
$$

Thus

$$
\begin{aligned}
\log \left\|s_{n}\right\| & \leqslant \log C+\log \left(\left\|s_{n-1}\right\|+2 C\right) \\
& \leqslant \log C(1+2 C)+\log \left\|s_{n-1}\right\| .
\end{aligned}
$$

It follows that for any $t \geqslant 1$ there exists an integer $n$ such that

$$
\log \left\|s_{n-1}\right\| \leqslant t \leqslant \log \left\|s_{n}\right\|
$$

and

$$
\log \left\|s_{n}\right\|-\log \left\|s_{n-1}\right\| \leqslant \log C(1+2 C) .
$$

Since $\phi$ and $\psi$ are Lipschitz, we conclude from inequality (*) and the above inequalities that $\sup _{0<t<\infty}|\phi(t)-\psi(t)|<\infty$, which was to be proved.

(b) If $E(\phi)$ and $E(\psi)$ are projectively equivalent then $F(x)$ and $G(a x)$ are equivalent for some $a \neq 0$. ( $F$ and $G$ are induced by $\varphi$ and $\psi$, respectively.) Define $G_{a}(x)=G(a x)$; then $E \oplus_{G_{a}} E$ is equivalent to $E(\theta)$, where

$$
\theta(t)=a \psi(t+\log 1 /|a|)-a \psi(\log 1 /|a|) .
$$

Hence

$$
\sup _{0<t<\infty}|\phi(t)-a \psi(t+\log 1 /|a|)+a \psi(\log 1 /|a|)|<\infty,
$$

and since $\phi$ and $\psi$ are Lipschitz,

$$
\sup _{0<t<\infty}|\phi(t)-a \psi(t)|<\infty
$$

This proves (b).

(c) is immediate from (a) and (b).

Definition 4.3. A pair of $F$-spaces $(X, Y)$ is said to split if every twisted sum of $X$ and $Y$ is trivial (i.e. every short exact sequence $0 \rightarrow X \rightarrow Z \rightarrow Y \rightarrow$ 0 splits).

Since the class $\mathcal{E}$ contains (many!) unbounded functions, an immediate consequence of Theorem 4.2 is:

COROLlary 4.4. Let $E$ be a solid quasi-normed FK-space such that no subsequence of $\left(e_{n}\right)$ is equivalent to the usual basis of $c_{0}$. Then $(E, E)$ fails to split.

Suppose that $E$ is a quasi-normed $F$-space with an unconditional basis, no subsequence of which is equivalent to a $c_{0}$-basis. Then $E$ is isomorphic to a space satisfying the assumptions of Corollary 4.4 , so $(E, E)$ fails to split. In particular,

COROllaRY 4.5. The pair $\left(l_{p}, l_{p}\right)$ fails to split for $0<p<\infty$. 
COROllary 4.6. For $0<p \leqslant 1$, there exists a quasi-normed $F$-space $Z$ which is not p-convex, but which has a subspace $Y$ isomorphic to $l_{p}$ with $Z / Y$ also isomorphic to $l_{p}$.

Corollaries 4.5 and 4.6 solve a problem raised in [4] for $0<p<1$. For $p=1$, these results are known; they are equivalent to results of Kalton [4], Ribe [7], and Roberts [8], which are constructions of a nonlocally convex $F$-space whose quotient by a one-dimensional subspace is a Banach space.

Theorem 2.6 of [4] implies that a twisted sum of two $B$-convex Banach spaces is (after renorming) a $B$-convex Banach space. This and Theorem 2.2 give

TheOREM 4.7. If $1<p<\infty$ and $\phi \in \mathcal{L}$, then $l_{p}(\phi)$ is isomorphic to a Banach space. In particular, $l_{p}(\phi)$ may be renormed so that it is projectively equivalent to an isometric twisted sum of $l_{p}$ and $l_{p}$.

Corollary 4.5 for $p=2$ and the above theorem give

COROLlARY 4.8. There is an isometric twisted sum of Banach spaces

$$
0 \rightarrow l_{2} \rightarrow Z \rightarrow l_{2} \rightarrow 0
$$

which does not split.

This was originally established by Enflo, Lindenstrauss, and Pisier [2]. Their approach was "local" in nature; our construction seems more "global" and somewhat simpler. The corresponding result for $p \neq 2$ also follows simply enough.

It is possible to extend these results slightly by using the ideas of Turpin [12]. We modify Turpin's approach slightly but not essentially.

Suppose $X$ is a (nontrivial) quasi-normed $F$-space. Then the galb $G(X)$ of $X$ is defined to be the space of all real sequences $\left\{a_{n}\right\}$ such that $\sum a_{n} x_{n}$ converges whenever $\left(x_{n}\right)$ is a bounded sequence in $X$. The space $G(X)$ is a quasi-normed $F$-space when equipped with the quasi-norm

$$
\left\|\left\{a_{n}\right\}\right\|=\sup _{m} \sup _{\left\|x_{i}\right\|<1}\left\|\sum_{i=1}^{m} a_{i} x_{i}\right\| .
$$

It is easily verified that $G(G(X))=G(X)$, and that $G(X)$ satisfies the hypotheses of Theorem 4.2 (see [12, 2.2 and 5.2]).

COROLlaRY 4.9. Suppose $X$ is a nontrivial quasi-normed $F$-space. Then there is a quasi-normed $F$-space $Z$ with a subspace $Y$ isomorphic to $G(X)$ such that $Z / Y$ is isomorphic to $G(X)$ but such that $G(Z) \subsetneq G(X)$.

Proof. From Corollary 4.4 there is a space $Z$ with a subspace $Y$ isomorphic to $G(X)$ with $Z / Y$ isomorphic to $G(X)$ and $Y$ not a direct summand of $Z$. Assume that $G(Z)=G(X)$. For each $n$ define $e_{n}$ in $G(X)$ by $e_{n}(k)=\delta_{n k}$. 
Let $q$ be the quotient map of $Z$ onto $G(X)$; then by the openness of $q$ we may choose $z_{n}$ in $Z$ with $q z_{n}=e_{n}$ and $\left\|z_{n}\right\| \leqslant M, M$ independent of $n$. Then since $G(Z)=G(X)$,

$$
\left\|\sum_{n=1}^{m} a_{n} z_{n}\right\| \leqslant M^{*}\left\|\left\{a_{1}, a_{2}, \ldots, a_{m}, 0, \ldots\right\}\right\|_{G(X)}
$$

where $M^{*}$ is independent of $m$ and $a_{1}, \ldots, a_{m}$. Thus we may define a continuous linear operator $T: G(X) \rightarrow Z$ such that $q T=i_{G(X)}$. (T is defined by $T e_{n}=z_{n}, n \in \mathbf{N}$.) This implies that $Y$ is a direct summand of $Z$; the proof is complete.

THEOREM 4.10. Suppose $E$ is a solid quasi-normed FK-space and $\phi \in \mathcal{L}$. Then $E(\phi)$ has a basis.

Proof. Suppose $\phi$ induces the quasi-linear map $F: E \rightarrow E$. Then $E(\phi)=$ $E \oplus_{F} E$. Let

$$
\begin{aligned}
u_{2 n-1} & =\left(e_{n}, 0\right), \\
u_{2 n} & =\left(0, e_{n}\right), \quad n \in \mathbf{N} .
\end{aligned}
$$

Then $\left(u_{n}\right)$ spans a dense subspace of $E(\phi)$; we verify that it is a basic sequence. It is enough to show that there is a constant $C^{*}$ such that if $p<q$ and $t_{1}, \ldots, t_{q}$ are in $\mathbf{R}$, then

$$
\left\|\sum_{i=1}^{p} t_{i} u_{i}\right\| \leqslant C^{*}\left\|\sum_{i=1}^{q} t_{i} u_{i}\right\| .
$$

Suppose first that $p$ is even, $p=2 n$. Then

$$
\begin{aligned}
\left\|\sum_{i=1}^{p} t_{i} u_{i}\right\| & =\left\|\sum_{i=1}^{n} t_{2 i-1} e_{i}-F\left(\sum_{i=1}^{n} t_{2 i} e_{i}\right)\right\|+\left\|\sum_{i=1}^{n} t_{2 i} e_{i}\right\| \\
& =\left\|\sum_{i=1}^{n}\left(t_{2 i-1}-t_{2 i} \phi\left(\log \alpha_{n}-\log \left|t_{2 i}\right|\right)\right) e_{i}\right\|+\alpha_{n},
\end{aligned}
$$

where $\alpha_{n}=\left\|\sum_{i=1}^{n} t_{2 i} e_{i}\right\|$. Now suppose $n<N$, and let $\alpha=\left\|\sum_{i=1}^{N} t_{2 i} e_{i}\right\|$. Then

$$
\begin{aligned}
\left\|\sum_{i=1}^{p} t_{i} u_{i}\right\|<C\left[\| \sum_{i=1}^{n}\left(t_{2 i-1}-\right.\right. & \left.t_{2 i} \phi\left(\log \alpha-\log \left|t_{2 i}\right|\right)\right) e_{i} \| \\
& \left.+L\left\|\sum_{i=1}^{n} t_{2 i}\left(\log \alpha-\log \alpha_{n}\right) e_{i}\right\|\right]+\alpha_{n},
\end{aligned}
$$

where $C$ is a modulus of concavity for $\|\cdot\|$ and $L$ is the Lipschitz constant of $\phi$. Hence 


$$
\begin{aligned}
\left\|\sum_{i=1}^{2 n} t_{i} u_{i}\right\|< & C\left\|\sum_{i=1}^{N}\left(t_{2 i-1}-t_{2 i} \phi\left(\log \alpha-\log \left|t_{2 i}\right| e_{i}\right)\right)\right\| \\
& +C L \log \left(\frac{\alpha}{\alpha_{n}}\right) \alpha_{n}+\alpha_{n} \\
\leqslant & C\left\|\sum_{i=1}^{N} t_{i} u_{i}\right\|+\left(\frac{C L}{e}+1\right) \alpha \\
\leqslant & \left(C+\frac{C L}{e}+1\right)\left\|\sum_{i=1}^{2 N} t_{i} u_{i}\right\| .
\end{aligned}
$$

(For the second inequality we have used that $|t \log t|<e^{-1}$ for $0<t<1$.)

Now if $p=2 n-1, n \leqslant N$, then

$$
\begin{aligned}
\left\|\sum_{i=1}^{p} t_{i} u_{i}\right\| & \leqslant C\left(\left\|\sum_{i=1}^{2 n} t_{i} u_{i}\right\|+\left\|t_{2 n} u_{2 n}\right\|\right) \\
& \leqslant C\left(C+\frac{C L}{e}+1\right)\left\|\sum_{i=1}^{2 N} t_{i} u_{i}\right\|+C\left|t_{2 n}\right|,
\end{aligned}
$$

where $C$ is a modulus of concavity for $\|\cdot\|$. However,

$$
\left|t_{2 n}\right|<\left\|\sum_{i=1}^{N} t_{2 i} e_{i}\right\|<\left\|\sum_{i=1}^{2 N} t_{i} u_{i}\right\|,
$$

so that

$$
\left\|\sum_{i=1}^{p} t_{i} u_{i}\right\|<\left(C^{2}+\frac{C^{2} L}{e}+2 C\right)\left\|\sum_{i=1}^{2 N} t_{i} u_{i}\right\| .
$$

Finally, since

$$
\left\|\sum_{i=1}^{2 k} t_{i} u_{i}\right\|<\left\|\sum_{i=1}^{2 k+1} t_{i} u_{i}\right\|
$$

for any $k$, the result follows-with $q$ equal either to $2 N$ or $2 N-1$.

5. The Banach spaces $l_{p}(\phi), 1<p<\infty$. For $1<p<\infty$ and $\phi \in \mathcal{L}$, the space $l_{p}(\phi)$ is a reflexive Banach space, since $l_{p}$ is reflexive. In this section we obtain some of its properties. We first identify the dual space of $l_{p}(\phi)$; then we study the structure of basic sequences in $l_{p}(\phi)$.

Toward our first objective: the adjoint of the short exact sequence

$$
0 \rightarrow l_{p} \rightarrow l_{p}(\phi) \rightarrow l_{p} \rightarrow 0
$$

is a short exact sequence

$$
0 \rightarrow l_{q} \rightarrow l_{p}(\phi)^{*} \rightarrow l_{q} \rightarrow 0,
$$

with $1 / p+1 / q=1$. It follows that $l_{p}(\phi)^{*}$ is a twisted sum $l_{q} \oplus_{G} l_{q}$, with the 
duality given by

$$
\langle(x, y),(w, z)\rangle=\langle y, w\rangle+\langle x, z\rangle
$$

for $x, y$ in $l_{p}$ and $w, z$ in $l_{q}$. The quasi-linear map $G$ can readily be identified:

THEOREM 5.1. The space $l_{p}(\phi)^{*}$ is equivalent to $l_{q}(\psi)$, where

$$
\psi(t)=-\phi\left((p-1)^{-1} t\right), \quad t>0 .
$$

Proof. The crucial step in the proof is the following lemma:

LEMMA 5.2. If $x_{1}, \ldots, x_{n}, y_{1}, \ldots, y_{n}$ are in $\mathbf{R}$ then

$$
\left|\sum_{i=1}^{n} x_{i} y_{i}\left(\log \frac{\left|x_{i}\right|}{\left|y_{i}\right|^{p-1}}\right)\right|<\frac{1}{e}\left((p-1)\|x\|_{q}^{q}+\|y\|_{p}^{p}\right) \text {. }
$$

(Here, $1<p<\infty, 1 / q+1 / p=1$, and $0 \log 0=0 \log \infty=0$.)

Proof. Let $J$ denote the set of indices in $\{1, \ldots, n\}$ such that $\left|x_{i}\right|<$ $\left|y_{i}\right|^{p-1}, i \in J$; and let $K$ denote the remaining indices. Then

$$
\begin{aligned}
\left|\sum_{i \in J} x_{i} y_{i}\left(\log \frac{\left|x_{i}\right|}{\left|y_{i}\right|^{p-1}}\right)\right| & =\left.\left|\sum_{i \in J} \frac{x_{i}}{\left|y_{i}\right|^{p-1}}\left(\log \frac{\left|x_{i}\right|}{\left|y_{i}\right|^{p-1}}\right) y_{i}\right| y_{i}\right|^{p-1} \mid \\
& <\frac{1}{e}\left(\|y\|_{p}^{p}\right)
\end{aligned}
$$

(using $|t \log | t||<1 / e,|t|<1$ ). Now,

$$
\left(\frac{\left|y_{i}\right|}{\left|x_{i}\right|^{q-1}}\right)^{p-1}=\frac{\left|y_{i}\right|^{p-1}}{\left|x_{i}\right|}
$$

so

$$
\begin{aligned}
\left|\sum_{i \in K} x_{i} y_{i}\left(\log \frac{\left|x_{i}\right|}{\left|y_{i}\right|^{p-1}}\right)\right| & =\left.\left|\sum_{i \in K} \frac{y_{i}}{\left|x_{i}\right|^{q-1}}\left(\log \frac{\left|x_{i}\right|}{\left|y_{i}\right|^{p-1}}\right) x_{i}\right| x_{i}\right|^{q-1} \mid \\
& <\frac{(p-1)}{e}\|x\|_{q \cdot}^{q}
\end{aligned}
$$

The inequality follows from combining these two estimates.

Proof of 5.1. We suppose that $\psi$ induces the quasi-linear map $H: l_{q} \rightarrow l_{q}$, so that for $x \in \mathbf{R}^{\infty}$,

$$
(H(x))_{n}=x_{n} \psi\left(-\log \frac{\left|x_{n}\right|}{\left\|x_{n}\right\|}\right) \text {. }
$$

Let $\mathbf{R}_{q}^{\infty}$ denote the space $\mathbf{R}^{\infty}$ with the $l_{q}$ norm, and define $T: R_{q}^{\infty} \oplus_{H} R_{q}^{\infty} \rightarrow$ $l_{p}(\phi)^{*}$ by $T(w, z)(x, y)=\langle y, w\rangle+\langle x, z\rangle$ for $x, y$ in $\mathbf{R}^{\infty}$. The inequalities which follow show that $T(w, z)$ defines a continuous linear functional and that $T$ is a bounded operator. 
Write

$$
\begin{aligned}
\langle y, w\rangle+\langle x, z\rangle= & \langle x-F(y), z\rangle+\langle F(y), z\rangle \\
& +\langle y, H(z)\rangle+\langle y, w-H(z)\rangle .
\end{aligned}
$$

By definition of $F, H$, and $\psi$,

$$
\begin{aligned}
\langle F(y), z\rangle & +\langle y, H(z)\rangle \mid \\
& =\left|\sum_{i=1}^{n} y_{i} z_{i}\left[\phi\left(\log \frac{\|y\|}{\left|y_{i}\right|}\right)+\psi\left(\log \frac{\|z\|}{\left|z_{i}\right|}\right)\right]\right| \\
& =\left|\sum_{i=1}^{n} y_{i} z_{i}\left[\phi\left(\log \frac{\|y\|}{\left|y_{i}\right|}\right)-\phi\left(\log \left(\frac{\|z\|}{\left|z_{i}\right|}\right)^{1 /(p-1)}\right)\right]\right| \\
& <L \sum_{i=1}^{n}\left|y_{i} z_{i} \log \left(\frac{\|y\|}{\left|y_{i}\right|} \cdot\left(\frac{\left|z_{i}\right|}{\|z\|}\right)^{1 /(p-1)}\right)\right| \\
& =\frac{L}{p-1} \sum_{i=1}^{n}\left|y_{i} z_{i} \log \left(\left(\frac{\|y\|}{\left|y_{i}\right|}\right)^{p-1} \cdot \frac{\left|z_{i}\right|}{\|z\|}\right)\right|
\end{aligned}
$$

Here, $L$ is the Lipschitz constant of $\phi$; and if any $y_{i}$ or $z_{i}$ is zero, the corresponding term in the sum is taken to be zero.

The last expression above equals

$$
\frac{L}{p-1} \sum_{i=1}^{n}\left|y_{i}\right| z_{i}^{*} \log \left(\frac{\|y\|^{p-1}\left|z_{i}^{*}\right|}{\left|y_{i}\right|^{p-1}\left\|z^{*}\right\|}\right)
$$

where

$$
z_{i}^{*}=\left|z_{i}\right| \operatorname{sgn} \log \left(\frac{\|y\|^{p-1}\left|z_{i}\right|}{\left|y_{i}\right|^{p-1}\|z\|}\right) .
$$

Now let $u_{i}=\left|y_{i}\right| /\|y\|$ and let $v_{i}=z_{i}^{*} /\left\|z^{*}\right\|$. Then

$$
\begin{aligned}
\langle F(y), z\rangle+\langle y, H(z)\rangle \mid & \leqslant \frac{L}{p-1}\|y\|\|z\| \sum_{i=1}^{n} u_{i} v_{i} \log \frac{\left|v_{i}\right|}{\left|u_{i}\right|^{p-1}} \\
& \leqslant \frac{L p}{(p-1) e}\|y\|\|z\|
\end{aligned}
$$

by Lemma 5.2.

Letting $K=\max \{L p /((p-1) e), 1\}$, we see from the above and Hölder's inequality that

$$
\begin{aligned}
\langle y, w\rangle+\langle x, z\rangle \mid & <K(\|x-F(y)\|\|z\|+\|y\|\|z\|+\|y\|\|w-H(z)\|) \\
& \leqslant K\|(x, y)\|\|(w, z)\| .
\end{aligned}
$$


This shows that $T(w, z)$ is continuous on $\mathbf{R}_{p}^{\infty} \oplus_{F} \mathbf{R}_{p}^{\infty}$ and hence that $T(w, z)$ defines an element of $l_{p}(\phi)^{*}$; it also shows that $T$ is a bounded operator from $\mathbf{R}_{q}^{\infty} \oplus_{H} \mathbf{R}_{q}^{\infty}$ into $l_{p}(\phi)^{*}$.

It is easy to check that $T$ induces an equivalence between the diagrams

$$
0 \rightarrow \mathbf{R}_{q}^{\infty} \rightarrow \mathbf{R}_{q}^{\infty} \oplus_{H} \mathbf{R}_{q}^{\infty} \rightarrow \mathbf{R}_{q}^{\infty} \rightarrow 0
$$

and

$$
0 \rightarrow \mathbf{R}_{q}^{\infty} \rightarrow l_{p}(\phi)^{*} \rightarrow \mathbf{R}_{q}^{\infty} \rightarrow 0 .
$$

The equivalence continues to hold when we pass to the completions of the spaces in the diagrams, and the proof of the theorem is complete.

We thank Haskell Rosenthal for simplifying the original proof of Lemma 5.2; the proof presented here is essentially due to him. We also thank Heinrich Lotz for pointing out an error in an earlier version of the deduction of Theorem 5.1 from Lemma 5.2.

We now turn to the structure of basic sequences in $l_{p}(\phi)$. For convenience, we suppose that $\phi$ is differentiable with bounded derivative. As before, the quasi-additive map $f$ induced by $\phi$ is $f(t)=t \phi(-\log t)$. Then

$$
f^{\prime}(t)=\phi(-\log t)-\phi^{\prime}(-\log t)
$$

so that $|f|$ is increasing near 0 provided $|\phi(t)| \rightarrow \infty$ as $t \rightarrow \infty$. Indeed, for $p>1, t^{-1}|f(t)|^{p}$ is increasing near 0 .

Notation. $l_{p,}$ is the space of all real sequences $\left(x_{n}\right)$ such that $\sum_{n=1}^{\infty}\left|f\left(\tau x_{n}\right)\right|^{p}$ $<\infty$ for all $\tau>0$.

This is the usual Orlicz sequence space $l_{M}$, where $M(t)=|f(t)|^{p}$ in a neighborhood of 0 and $M$ is extended so as to be an increasing function. The space $l_{M}$ is a Banach space, since $t^{-1} M(t)$ is increasing near 0 and $M$ is therefore equivalent to a convex Orlicz function.

LEMMA 5.3. Let $\left(v_{n}\right)$ be a normalized block basic sequence in $l_{p}$ and let $w_{n}=\left(F\left(v_{n}\right), v_{n}\right)$ in $l_{p}(\phi)$. Then

(a) the sequence $\left(w_{n}\right)$ is a basic sequence;

(b) if $\lim _{t \rightarrow \infty} \phi^{\prime}(t)=0$ monotonically, then $\left(w_{n}\right)$ has a subsequence equivalent either to the usual basis of $l_{p}$ or the usual basis of $l_{f, p}$;

(c) if $\phi(t)=c t, c \neq 0$, then $\left(w_{n}\right)$ is equivalent to the usual basis of $l_{f, p}$.

Proof. (a) The sequence $\left(w_{n}\right)$ is block basic with respect to the basis constructed in Corollary 4.9, and hence is a basic sequence.

(b) Assume first that $\sum_{n=1}^{\infty} t_{n} w_{n}$ converges. Then $\sum_{n=1}^{\infty} t_{n} v_{n}$ converges, so $\sum_{n=1}^{\infty}\left|t_{n}\right|^{p}<\infty$.

Now suppose $\sum_{n=1}^{\infty}\left|t_{n}\right|^{p}<\infty$. Let

$$
\sigma_{N}=\left(\sum_{n=1}^{N}\left|t_{n}\right|^{p}\right)^{1 / p}, \quad n \in \mathbf{N}
$$


and

$$
\sigma=\left(\sum_{n=1}^{\infty}\left|t_{n}\right|^{p}\right)^{1 / p}
$$

Assume that $v_{n}=\sum_{k=l_{n-1}+1}^{l_{n}} v_{n}(k) e_{k}$, where $\left(l_{n}\right)$ is an increasing sequence of integers. Then

$$
\begin{array}{r}
\left\|\sum_{n=1}^{N} t_{n} w_{n}\right\|=\sigma_{N}+\left(\sum_{n=1}^{N} \sum_{k=l_{n-1}+1}^{l_{n}}\left|t_{n}\right|^{p}\left|v_{n}(k)\right|^{p} \mid \phi\left(\log \frac{\sigma_{N}}{\left|t_{n} v_{n}(k)\right|}\right)\right. \\
\left.-\left.\phi\left(\log \frac{1}{\left|v_{n}(k)\right|}\right)\right|^{p}\right)^{1 / p} .
\end{array}
$$

Since $\sigma_{N} \rightarrow \sigma$ and $\phi$ is Lipschitz, it is easy to see that if

$$
\lim _{N \rightarrow \infty}\left\|\sum_{n=1}^{N} t_{n} w_{n}\right\|
$$

exists, the limit must be

$$
\sigma+\left(\sum_{n=1}^{\infty} \sum_{k=l_{n-1}+1}^{l_{n}}\left|t_{n}\right|^{p}\left|v_{n}(k)\right|^{p}\left|\phi\left(\log \frac{\sigma}{\left|t_{n} v_{n}(k)\right|}\right)-\phi\left(\log \frac{1}{\left|v_{n}(k)\right|}\right)\right|^{p}\right)^{1 / p} .
$$

Hence, since $\left(w_{n}\right)$ is boundedly complete $\left(l_{p}(\phi)\right.$ is reflexive), $\sum_{n=1}^{\infty} t_{n} w_{n}$ converges if and only if the expression (**) is finite.

By assumption, $\lim _{t \rightarrow \infty} \phi^{\prime}(t)=0$ monotonically, so that

$$
\begin{aligned}
\mid \phi\left(\log \frac{\sigma}{\left|t_{n} v_{n}(k)\right|}\right)- & \phi\left(\log \frac{1}{\left|v_{n}(k)\right|}\right) \mid \\
& <\left|\phi\left(\log \frac{\sigma}{\left|t_{n}\right|}\right)-\phi(0)\right|=\left|\phi\left(\log \frac{\sigma}{\left|t_{n}\right|}\right)\right| .
\end{aligned}
$$

Thus $\sum_{n=1}^{\infty} t_{n} w_{n}$ converges provided

$$
\sum_{n=1}^{\infty}\left|t_{n}\right|^{p}\left|\phi\left(\log \frac{\sigma}{\left|t_{n}\right|}\right)\right|^{p}<\infty
$$

or, since $\phi$ is Lipschitz, provided

$$
\sum_{n=1}^{\infty}\left|f\left(t_{n}\right)\right|^{p}<\infty
$$

We now have two cases.

(i) If $a_{n}=\max \left\{\left|v_{n}(k)\right|: l_{n-1}+1<k<l_{n}\right\}>\varepsilon>0$ for all $n$, we show that the finiteness of $(* *)$ implies the finiteness of $(* * *)$. (Then $\left(w_{n}\right)$ is equivalent to the usual basis of $l_{f, p}$.) In this case, the expression (**) is greater than 


$$
\begin{aligned}
\varepsilon\left(\sum_{n=1}^{\infty}\left|t_{n}\right|^{p}\left|\phi\left(\log \frac{\sigma}{\left|t_{n} \varepsilon\right|}\right)-\phi\left(\log \frac{1}{\varepsilon}\right)\right|^{p}\right)^{1 / p} \\
\qquad \varepsilon\left(\sum_{n=1}^{\infty}\left|t_{n}\right|^{p}\left|\phi\left(\log \frac{\sigma}{\left|t_{n}\right|}\right)\right|^{p}\right)^{1 / p}-\varepsilon(L+1) \phi(\log 1 / \varepsilon) \sigma ;
\end{aligned}
$$

this implies (***) is finite. ( $L$ is the Lipschitz constant of $\phi$.

(ii) If $\inf _{n} a_{n}=0$, there is a subsequence $\left(v_{k_{n}}\right)$ of $\left(v_{n}\right)$ such that $\left|\phi^{\prime}(t)\right|<2^{-n}$ for $t>\log \left(1 / a_{k_{n}}\right)$. For ease of notation we assume $k_{n}=n$. Then

$$
\left|\phi\left(\log \frac{\sigma}{\left|t_{n} v_{n}(k)\right|}\right)-\phi\left(\log \frac{1}{\left|v_{n}(k)\right|}\right)\right| \leqslant 2^{-n} \log \frac{\sigma}{\left|t_{n}\right|} .
$$

Now, using that $|t \log \sigma / t| \leqslant \sigma e^{-1}$ for $0 \leqslant t \leqslant \sigma$, we have that the expression (**) is bounded above by

$$
\sigma+\left(\sum_{n=1}^{\infty} 2^{-n p}\left|t_{n}\right|^{p}\left(\log \frac{\sigma}{\left|t_{n}\right|}\right)^{p}\right)^{1 / p}<\sigma+\sigma e^{-1}\left(\sum_{n=1}^{\infty} 2^{-n p}\right)^{1 / p}<\infty .
$$

Thus in this case, $\left(v_{k_{n}}\right)$ is equivalent to the usual basis of $l_{p}$. The alternatives (i) and (ii) imply (b).

For the proof of (c), note that if $\phi(t)=c t, c \neq 0$, then the expression (**) equals

$$
\sigma+|c|\left(\sum_{n=1}^{\infty}\left|t_{n}\right|^{p}\left|\log \frac{\sigma}{\left|t_{n}\right|}\right|^{p}\right)^{1 / p}
$$

thus $\left(w_{n}\right)$ is equivalent to the usual basis of $l_{f, p}$.

TheOREM 5.4. Suppose that either $\lim _{t \rightarrow \infty} \phi^{\prime}(t)=0$ monotonically or $\phi(t)=$ $c t, c \neq 0$. Then every normalized basic sequence in $l_{p}(\phi)$ has a subsequence equivalent either to the usual basis of $l_{p}$ or to the usual basis of $l_{f_{p}}$.

Proof. Suppose that $w_{n}=\left(u_{n}, v_{n}\right)$ is a normalized basic sequence in $l_{p}(\phi)$.

If $\left\|v_{n}\right\| \rightarrow 0$, then

$$
\left\|\left(u_{n}, v_{n}\right)-\left(u_{n}-F\left(v_{n}\right), 0\right)\right\|=\left\|\left(F\left(v_{n}\right), v_{n}\right)\right\| \rightarrow 0 .
$$

Also, $\left(u_{n}-F\left(v_{n}\right), 0\right)$ is in $j\left(l_{p}\right)$. Hence, by a standard perturbation of bases argument, $\left(w_{n}\right)$ has a subsequence equivalent to a normalized basic sequence in $l_{p}$. From this the result follows easily.

Now suppose $\left\|v_{n}\right\| \nrightarrow 0$. Then by passing to a subsequence we may assume that $\left\|v_{n}\right\| \geqslant \varepsilon>0$ for all $n$. From the fact that $\left(w_{n}\right)$ is shrinking and the characterization of $l_{p}(\phi)^{*}$, it follows that $v_{n} \rightarrow 0$ weakly. Again, by passing to a subsequence, we may suppose that there is a block basic sequence $\left(y_{n}\right)$ in $l_{p}$ with $\left\|v_{n}-y_{n}\right\| \leqslant 2^{-n}$.

By passing to a further subsequence and using Lemma 5.3, we may suppose 
that $\left(F\left(y_{n}\right), y_{n}\right)$ is a basic sequence equivalent either to the usual basis of $l_{p}$ or to the usual basis of $l_{f, p}$. Let $z_{n}=\left(F\left(y_{n}\right), y_{n}\right)$; then $z_{n} \rightarrow 0$ weakly.

If $\left\|w_{n}-z_{n}\right\| \rightarrow 0$, a subsequence of $\left(w_{n}\right)$ is equivalent to a subsequence of $z_{n}$; an application of Lemma 5.3 completes the argument in this case.

If $\left\|w_{n}-z_{n}\right\| \nrightarrow 0$, by passing to a further subsequence we may assume that $\left(w_{n}-z_{n}\right)$ is a basic sequence. However, $w_{n}-z_{n}=\left(u_{n}-F\left(y_{n}\right), v_{n}-y_{n}\right)$, and $\left\|v_{n}-y_{n}\right\| \rightarrow 0$. Hence by the very first part of the argument we may assume that $\left(w_{n}-z_{n}\right)$ is equivalent to the usual basis of $l_{p}$.

Thus if $\sum_{n=1}^{\infty} t_{n} w_{n}$ converges, then $\sum_{n=1}^{\infty} t_{n} v_{n}$ converges, and hence $\sum_{n=1}^{\infty}\left|t_{n}\right|^{p}<\infty$. Then $\sum_{n=1}^{\infty} t_{n}\left(w_{n}-z_{n}\right)$ and hence $\sum_{n=1}^{\infty} t_{n} z_{n}$ converge. Conversely, if $\sum_{n=1}^{\infty} t_{n} z_{n}$ converges, then since $\sum_{n=1}^{\infty} t_{n} y_{n}$ converges, certainly $\sum_{n=1}^{\infty}\left|t_{n}\right|^{p}<\infty$; and we may reverse the reasoning.

The converse is trivial by using the sequences $\left(e_{n}, 0\right)$ and $\left(0, e_{n}\right)$. This completes the proof of Theorem 5.4.

COROLlary 5.5. If we take

$$
\begin{aligned}
\phi_{r}(t) & =t, \quad 0 \leqslant t \leqslant 1, \\
& =t^{r}, \quad 1<t<\infty,
\end{aligned}
$$

then the spaces $l_{p}\left(\phi_{r}\right)(0<r \leqslant 1)$ are mutually nonisomorphic.

This is immediate from Theorem 5.4, since $l_{p}\left(\phi_{r}\right)$ contains $l_{f_{r}, p}$, where $f_{r}$ is induced by $\phi_{r}$. Since $f_{r}$ and $f_{s}$ are not equivalent at 0 for $r \neq s$ (see $\left.[5,4 . a .5]\right)$, the result follows.

Strictly speaking, in order to apply Theorem 5.4 we should first smooth $\phi_{r}$.

CoRollary 5.6. Let $\phi(t)=c t(c \neq 0)$ and let $\left(w_{n}\right)=\left(u_{n}, v_{n}\right)$ be a normalized basic sequence in $l_{p}(\phi)$ with inf $\left\|v_{n}\right\|>0$. Then $\left(w_{n}\right)$ has a subsequence equivalent to the usual basis of $l_{f, p}$.

Proof. This follows from an examination of the proof of Theorem 5.4. By Lemma 5.3(c), the sequence $\left(z_{n}\right)$ in the proof of Theorem 5.4 is equivalent to the usual basis of $l_{f, p}$.

6. The Banach spaces $Z_{p}, 1<p<\infty$. In this section we examine more carefully the extremal example of the class of spaces we have constructed. For fixed $p$, the spaces $l_{p}(\phi)$ obtained by taking $\phi(t)=c t, c \neq 0$, are projectively equivalent twisted sums and hence isomorphic as Banach spaces.

Notation. $Z_{p}$ denotes the (isomorphism class of) the Banach space $l_{p}(\phi)$, $\phi(t)=c t, t \neq 0$.

The next theorem summarizes the properties of $Z_{p}$ obtained so far.

TheOREM 6.1. For $1<p<\infty, Z_{p}$ is a reflexive Banach space with a basis. $Z_{p}$ may be normed in such a way that it has a closed subspace $M$ isometric to $l_{p}$ with $Z_{p} / M$ also isometric to $l_{p}$. Further, $Z_{p}^{*}$ is isometric to $Z_{q}$, where $1 / p+$ $1 / q=1$. However, $Z_{p}$ is not isomorphic to $l_{p}$. 
We claim that $Z_{p}$ is, in certain senses, an extremal solution to the threespace problem for $l_{p}$. In this section we will make the meaning of this more precise.

Let $\left(r_{i}\right)$ denote the sequence of Rademacher functions. For a Banach space $X$ and for $0<p \leqslant 2$, the number $a_{n, p}(X)$ is defined to be the least constant $a$ such that

$$
\left(\int_{0}^{1}\left\|\sum_{i=1}^{n} r_{i}(t) x_{i}\right\|^{p} d t\right)^{1 / p} \leqslant a\left(\sum_{i=1}^{n}\left\|x_{i}\right\|^{p}\right)^{1 / p}
$$

for all $x_{1}, \ldots, x_{n}$ in $X$. The space $X$ is of type $p$ if $\sup _{n} a_{n, p}(X)<\infty$. Enflo, Lindenstrauss, and Pisier [2, Theorem 3] show that if $X$ is a twisted sum of two Banach spaces of type 2 , then $a_{n, 2}(X)=O\left((\log n)^{\alpha}\right)$ for some constant $\alpha$. Our next result sharpens this somewhat.

THEOREM 6.2. (a) Let $X$ be a twisted sum of two Banach spaces which are isomorphic to subspaces of $L_{p}(1<p \leqslant 2)$. Then $a_{n, p}(X)=O(\log n)$.

(b) There is a constant $c>0$ such that $a_{n p}\left(Z_{p}\right) \geqslant c \log n(1<p<2)$.

Proof. The space $X$ is isomorphic to an isometric twisted sum of subspaces $Y$ and $Z$ of $L_{p}$. Then $a_{n, p}(Y)=a_{n, p}(Z)=1$ for all $n$. Now the proof of Theorem 3 of [2] shows that if $p=2, a_{n^{2} p}(X) \leqslant 2 a_{n, p}(X)+1, n=1,2, \ldots$ The same inequality for $1<p<2$ follows from identical reasoning.

Thus if $a_{2, p}(X)=k$, then

$$
a_{2^{2 n}, p}(X) \leqslant 2^{n} k+2^{n}-1 \leqslant(k+1) 2^{n}, \quad n=1,2, \ldots
$$

Since $a_{n, p}(X)$ is increasing in $n$ we easily obtain (a).

(b) Take $\phi(t)-t$; then $Z_{p}$ is isomorphic to $l_{p}(\phi)$. In $l_{p}(\phi)$ let $u_{n}=\left(0, e_{n}\right)$. Then $\left\|u_{n}\right\|=1$ and for $0 \leqslant t \leqslant 1$,

$$
\begin{aligned}
\left\|\sum_{i=1}^{n} r_{i}(t) u_{i}\right\| & =\left\|F\left(\sum_{i=1}^{n} r_{i}(t) e_{i}\right)\right\|+\left\|\sum_{i=1}^{n} r_{i}(t) e_{i}\right\| \\
& =\left\|\sum_{i=1}^{n} r_{i}(t) \log \left(n^{1 / p}\right) e_{i}\right\|+\left\|\sum_{i=1}^{n} r_{i}(t) e_{i}\right\| \\
& =\left(\frac{1}{p}(\log n)+1\right) n^{1 / p}
\end{aligned}
$$

while $\left(\sum_{i=1}^{n}\left\|u_{i}\right\|^{p}\right)^{1 / p}=n^{1 / p}$. Of course this calculation used the given quasinorm on $l_{p}(\phi)$, and we only conclude that for the norm on $Z_{p}, a_{n p}\left(Z_{p}\right)>$ $c \log n$ for some $c>0$. This completes the proof.

In a similar vein we have 
THEOREM 6.3. For $1<p<\infty$ there is a sequence of $2 n$-dimensional normed spaces $E_{n}$ satisfying

(a) each $E_{n}$ has an n-dimensional subspace $F_{n}$ such that $F_{n}$ and $E_{n} / F_{n}$ are isometric to $l_{p}^{n}$;

(b) if $\gamma_{n}$ denotes the least norm of a projection of $E_{n}$ onto $F_{n}$, then $\gamma_{n}>$ $c \log n$ for some $c=c(p)>0$.

Proof. By duality it is enough to consider the case $1<p<2$. Let $\phi(t)=$ $t$; in $l_{p}(\phi)=l_{p} \oplus_{F} l_{p}$, let $E_{n}$ be the linear span of $\left(0, e_{j}\right),\left(e_{j}, 0\right)(1<j<n)$ and let $F_{n}$ be the linear span of $\left(e_{j}, 0\right)(1<j<n)$.

Then $F_{n}$ is isometric to $l_{p}^{n}$ and $E_{n} / F_{n}$ is isometric to $l_{p}^{n}$, although $E_{n}$ as a subspace of $l_{p}(\phi)$ is only a quasi-normed space. However, $l_{p}(\phi)$ is isomorphic to the Banach space $Z_{p}$, and so we may consider $E_{n}$ and $F_{n}$ as subspaces of $Z_{p}$ with its (Banach space) norm. Thus for some $K=K(p)<\infty, F_{n}$ and $E_{n} / F_{n}$ are $K$-isomorphic to $l_{p}^{n}$. Now by using the technique of Proposition 2.2, the (normed) spaces $E_{n}$ may be uniformly renormed so that $F_{n}$ and $E_{n} / F_{n}$ are isometric to $l_{p}^{n}$. It therefore suffices to prove the result using the original quasi-norm on $l_{p}(\phi)$.

Suppose that $P: E_{n} \rightarrow F_{n}$ is a projection; then, for $x$ in $l_{p}^{n}$,

$$
\|P(F(x), x)\| \leqslant\|P\| \cdot\|x\|
$$

where $F: l_{p} \rightarrow l_{p}$ is the induced quasi-linear map. Since $P$ is a projection, it has the form $P(x, y)=(x+A y, 0)$, where $A: l_{p}^{n} \rightarrow l_{p}^{n}$ is a linear map. Hence

$$
\|F(x)+A x\| \leqslant\|P\| \cdot\|x\|
$$

and so $\left\|A e_{j}\right\| \leqslant\|P\|$ for $j \leqslant n$ (since $F\left(e_{j}\right)=0$ ). Now

$$
\begin{aligned}
\iint_{0}^{1} \| F\left(\sum_{j=1}^{n} r_{j}(t) e_{j}\right) & \left.+\sum_{j=1}^{n} r_{j}(t) A e_{j} \|^{p} d t\right]^{1 / p} \\
& \leqslant\|P\|\left\|\sum_{j=1}^{n} r_{j}(t) e_{j}\right\|=\|P\| n^{1 / p} .
\end{aligned}
$$

However,

$$
\begin{aligned}
\iint_{0}^{1} \| F\left(\sum_{j=1}^{n} r_{j}(t) e_{j}\right) & \left.+\sum_{j=1}^{n} r_{j}(t) A e_{j} \|^{p} d t\right]^{1 / p} \\
& \geqslant\left[\int_{0}^{1}\left\|F\left(\sum_{j=1}^{n} r_{j}(t) e_{j}\right)\right\|^{p} d t\right]^{1 / p}-\left(\int_{0}^{1}\left\|\sum_{j=1}^{n} r_{j}(t) A e_{j}\right\|^{p} d t\right)^{1 / p} \\
& \geqslant \frac{1}{p}(\log n) n^{1 / p}-\left(\sum_{j=1}^{n}\left\|A e_{j}\right\|^{p}\right)^{1 / p} \\
& \geqslant n^{1 / p}((1 / p) \log n-\|P\|) .
\end{aligned}
$$


Hence $(1 / p) \log n-\|P\| \leqslant\|P\|$, i.e., $\|P\| \geqslant(1 / 2 p) \log n$.

This completes the proof.

REMARKs. (1) The construction of a sequence of spaces $E_{n}$ as above with $\gamma_{n} \rightarrow \infty$ is the essential part of the solution to the three-space problem given by Enflo, Lindenstrauss, and Pisier in [2]. In their construction, $\gamma_{n}>$ $c(\log n)^{1 / 2}$; the result above improves this estimate slightly.

A theorem of Figiel, Lindenstrauss, and Milman [2a, Theorem 6.5] shows that $\gamma_{n}$ must be $O(\log n)^{2}$. Our Theorem 6.2(a) obtains the estimate in their proof: $a_{n, 2}(X)=O(\log n)$.

(2) We conjecture that the rate of growth $\gamma_{n} \sim \log n$ is the best possible here. That is, there exists $d>0$ such that whenever $\operatorname{dim} E_{n}=2 n$ and $F_{n}$ is an $n$-dimensional subspace of $E_{n}$ with $F_{n} \cong l_{p}^{n} \cong E_{n} / F_{n}$, then there is a projection of $E_{n}$ onto $F_{n}$ of norm at most $d(\log n+1)$.

It is perhaps worth noting that the Radamacher averaging technique used in Theorem 6.2 can be used to show directly that the pair $\left(l_{p}, l_{p}\right)$ does not split for $1<p \leqslant 2$.

Alternate PROOF OF 4.5 FOR $1<p \leqslant 2$. Let $\phi(t)=t, t \in \mathbf{R}$, and let $F$ be the induced quasi-linear map. If $l_{p}(\phi)$ is trivial, there are a linear map $A$ : $\mathbf{R}^{\infty} \rightarrow l_{p}$ and a constant $M$ such that $\|F(x)-A x\| \leqslant M\|x\|, x \in \mathbf{R}^{\infty}$. Since $F\left(e_{i}\right)=0$ for all $i$, we have that $\left\|A e_{i}\right\| \leqslant M$ for all $i$.

Now for $0 \leqslant t \leqslant 1$ and $n \in \mathbf{N}$, let $x_{n}(t)=\sum_{i=1}^{n} r_{i}(t) e_{i}$. We have that

$$
\left\|x_{n}(t)\right\|=n^{1 / p} \text { and }\left\|F\left(x_{n}(t)\right)\right\|=\frac{1}{p}(\log n) n^{1 / p} \text {. }
$$

Now,

$$
\left\|A\left(x_{n}(t)\right)\right\| \geqslant(1 / p)(\log n) n^{1 / p}-M n^{1 / p} .
$$

On the other hand,

$$
\begin{aligned}
\left(\int_{0}^{1}\left\|A\left(x_{n}(t)\right)\right\|^{p} d t\right)^{1 / p} & =\left(\int_{0}^{1}\left\|\sum_{i=1}^{n} r_{i}(t) A e_{i}\right\|^{p} d t\right)^{1 / p} \\
& \leqslant M^{\prime} M n^{1 / p}
\end{aligned}
$$

(again, as in Theorem 6.2, using that $l_{p}$ is of type $p$ for $p<2$ ). Inequalities (1) and (2) cannot both hold for large $n$, and the argument is complete. (Note that the argument is valid for $0<p \leqslant 1$ as well!)

To conclude the paper we describe another sense in which $Z_{p}$ is an extremal twisted sum of $l_{p}$ 's.

We need two definitions. If $X$ and $Y$ are Banach spaces, an operator $S$ : $X \rightarrow Y$ is strictly singular if for any infinite-dimensional Banach space $M$ and any operator $A: M \rightarrow X$, the composition $S A$ fails to be an isomorphism. Dually, $S$ is strictly co-singular if for any infinite-dimensional Banach space $N$ and any operator $B: Y \rightarrow N$, the composition $B S$ fails to be an open map. It 
is easy to construct a quotient map which is strictly singular (e.g., an operator from $l_{1}$ onto $l_{2}$ ), or dually, an isomorphism which is strictly co-singular.

However, $0 \rightarrow l_{p} \stackrel{j}{\rightarrow} Z_{p} \stackrel{q}{\rightarrow} l_{p} \rightarrow 0$ provides an example of a short exact sequence such that $j$ is strictly co-singular and $q$ is strictly singular. We know of no other example of this.

THEOREM 6.4. For $1<p<\infty$, the map $j: l_{p} \rightarrow Z_{p}$ is strictly co-singular and the map $q: Z_{p} \rightarrow l_{p}$ is strictly singular.

Proof. By Theorem 5.1 the adjoint of the short exact sequence

$$
0 \rightarrow l_{p} \rightarrow Z_{p} \rightarrow l_{p} \rightarrow 0
$$

is (projectively equivalent to) the short exact sequence

$$
0 \rightarrow l_{q} \rightarrow Z_{q} \rightarrow l_{q} \rightarrow 0,
$$

$1 / p+1 / q=1$. So by duality we only need show that $q: Z_{p} \rightarrow l_{p}$ is strictly singular. Assume not; then (since $l_{p}$ is hereditarily $l_{p}$ ), there is a normalized basic sequence $\left(w_{n}\right)$ in $Z_{p}$ equivalent to the usual $l_{p}$ basis such that $\left\|q w_{n}\right\|>\varepsilon$ $>0$ for all $n$. By Corollary 5.6 this is impossible, and the proof is complete.

We now examine further the strictly singular operators on $Z_{p}$. For each of the known prime Banach spaces $X=l_{p}(1<p<\infty)$ or $c_{0}$, the following property holds: if $T: X \rightarrow Y$ is not strictly singular, there is a subspace $X_{0}$ of $X$ isomorphic to $X$ such that $T$ is an isomorphism on $X_{0}$. For $l_{p}(1<p<\infty)$ and $c_{0}$ this is well known. For $l_{\infty}$, this follows from a theorem of Rosenthal [10, Theorem 3.7]: if $T: l_{\infty} \rightarrow Y$ is not an isomorphism on any subspace isomorphic to $l_{\infty}$, then $T$ is weakly compact. Since $T$ has the Dunford-Pettis property, $T$ is not an isomorphism on any infinite-dimensional subspace of $l_{\infty}$. It turns out that $Z_{p}$ has a similar but weaker property.

THEOREM 6.5. If $T: Z_{p} \rightarrow Z_{p}$ is not strictly singular, there is a subspace $W$ of $Z_{p}$ which is isomorphic to $Z_{p}$ such that $T$ is an isomorphism on $W$.

PROOF. Since $Z_{p}$ is a twisted sum of $l_{p}$ 's it is hereditarily $l_{p}$; hence there is a subspace $M$ of $Z_{p}, M$ isomorphic to $l_{p}$, such that $T \mid M$ is an isomorphism. Let $\left(u_{n}\right)$ be a basis of $M$ equivalent to the usual $l_{p}$ basis. Then by Corollary 5.6, $\left\|q u_{n}\right\| \rightarrow 0$ and $\left\|q T u_{n}\right\| \rightarrow 0$. By the first part of the proof of Theorem 5.4, we may assume (by passing to subsequences) that there are basic sequences $\left(v_{n}^{\prime}\right)$ and $\left(w_{n}^{\prime}\right)$ in $j\left(l_{p}\right)$ such that

$$
\left\|u_{n}-v_{n}^{\prime}\right\| \rightarrow 0 \quad \text { and } \quad\left\|T u_{n}-w_{n}^{\prime}\right\| \rightarrow 0 .
$$

Passing to subsequences again, we may assume that there are block basic sequences $\left(v_{n}\right)$ and $\left(w_{n}\right)$ in $l_{p}$ such that

$$
\left\|u_{n}-j v_{n}\right\| \leqslant 2^{-n}, \quad\left\|T u_{n}-j w_{n}\right\| \leqslant 2^{-n}, \quad n \in \mathbf{N} .
$$


Now for large enough $N$ we may construct bounded operators $A: Z_{p} \rightarrow Z_{p}$ and $B: Z_{p} \rightarrow Z_{p}$ with $\|A\| \leqslant \frac{1}{2},\|B\| \leqslant \frac{1}{2}$, and such that

$$
\begin{array}{ll}
A\left(j v_{n}\right)=u_{n}-j v_{n}, & n \geqslant N, \\
B\left(T u_{n}\right)=j w_{n}-T u_{n}, & n \geqslant N .
\end{array}
$$

(The operators $A$ and $B$ are easily defined in terms of Hahn-Banach extensions of the coefficient functionals for the bases $\left(j v_{n}\right)$ and $\left(T u_{n}\right)$, respectively.) Then

$$
(I+B) T(I+A) j v_{n}=j w_{n}, \quad n \geqslant N .
$$

Let $S=(I+B) T(I+A)$. Since $I+B$ and $I+A$ are invertible, it suffices to prove the result for $S$.

Now define $V: \mathbf{R}^{\infty} \oplus \mathbf{R}^{\infty} \rightarrow \mathbf{R}^{\infty} \oplus \mathbf{R}^{\infty}$ by

$$
V\left(e_{n}, 0\right)=\left(v_{n}, 0\right)=j\left(v_{n}\right), \quad V\left(0, e_{n}\right)=\left(F\left(v_{n}\right), v_{n}\right)
$$

and extending linearly. We will show that $V$ is an isomorphism; then after extension $V$ may be regarded as an isomorphism on $Z_{p}$. For $x, y$ in $\mathbf{R}^{\infty}$,

$$
V(x, y)=\left(\sum x_{n} v_{n}+\sum y_{n} F\left(v_{n}\right), \sum y_{n} v_{n}\right)
$$

where the sums are finite. Hence (using the original quasi-norm on $Z_{p}$ ),

$$
\|V(x, y)\|=\left\|\sum x_{n} v_{n}+\sum y_{n} F\left(v_{n}\right)-F\left(\sum y_{n} v_{n}\right)\right\|+\left\|\sum y_{n} v_{n}\right\| .
$$

If $v_{n}=\sum_{\eta_{n-1}+1}^{l_{n}} v_{n}(k) e_{k}$, then

$$
\begin{aligned}
\sum y_{n} F\left(v_{n}\right)-F\left(\sum y_{n} v_{n}\right) & =\sum_{n} \sum_{k=l_{n-1}+1}^{l_{n}} y_{n} v_{n}(k) \log \left(\frac{\left\|v_{n}\right\|\left|y_{n}\right|}{\left\|\sum y_{n} v_{n}\right\|}\right) e_{k} \\
& =\sum_{n} y_{n} \log \left(\frac{\left\|v_{n}\right\|\left|y_{n}\right|}{\left\|\sum y_{n} v_{n}\right\|}\right) v_{n} .
\end{aligned}
$$

Now there exist positive constants $\alpha, \beta$ so that $\alpha \leqslant\left\|v_{n}\right\|<\beta$, $n=$ $1,2 \ldots$ Hence

$$
\begin{aligned}
\| \sum x_{n} v_{n}+ & \left.\sum y_{n} F\left(v_{n}\right)-F\left(\sum y_{n} v_{n}\right) \|<\beta\left(\sum_{n} \mid x_{n}+y_{n} \log \frac{\left\|v_{n}\right\|\left|y_{n}\right|}{\left\|\sum y_{n} v_{n}\right\|}\right)^{p}\right)^{1 / p} \\
& \leqslant \beta\left(\sum_{n}\left|x_{n}+y_{n} \log \frac{\left|y_{n}\right|}{\|y\|}\right|^{p}+\beta\left(\sum_{n}\left|y_{n}\right|^{p}\left|\log \frac{\left\|v_{n}\right\|\|y\|}{\left\|\sum y_{n} v_{n}\right\|}\right|^{p}\right]^{1 / p}\right. \\
& \leqslant \beta\|x-F(y)\|+\beta\left(\log \frac{\beta}{\alpha}\right)\|y\| .
\end{aligned}
$$


Hence $\|V\| \leqslant \beta+\beta \log (\beta / \alpha)$. For the other direction,

$$
\begin{aligned}
\left\|\sum x_{n} v_{n}+\sum y_{n} F\left(v_{n}\right)-F\left(\sum y_{n} v_{n}\right)\right\| & \geqslant \alpha\left(\sum_{n}\left|x_{n}+y_{n} \log \frac{\left\|v_{n}\right\|\left|y_{n}\right|}{\left\|\sum y_{n} v_{n}\right\|}\right|^{p}\right)^{1 / p} \\
& \geqslant \alpha\|x-F(y)\|-\alpha\left(\log \frac{\beta}{\alpha}\right)\|y\| .
\end{aligned}
$$

Thus,

$$
\|V(x, y)\| \geqslant \max (\alpha\|x-F(y)\|-\alpha(\log (\beta / \alpha))\|y\|, \alpha\|y\|) .
$$

If $\|x-F(y)\| \geqslant 2(\log (\beta / \alpha))\|y\|$, then

$$
\|V(x, y)\| \geqslant \frac{\alpha}{2}\|x-F(y)\| \geqslant\left(\frac{\alpha \log (\beta / \alpha)}{1+2 \log (\beta / \alpha)}\right)\|(x, y)\| ;
$$

if $\|x-F(y)\| \leqslant 2(\log (\beta / \alpha))\|y\|$, then

$$
\|V(x, y)\| \geqslant\|y\| \geqslant(1+2 \log (\beta / \alpha))^{-1}\|(x, y)\| .
$$

Thus, $V$ is an isomorphism.

Similarly, if we let

$$
W\left(e_{n}, 0\right)=\left(w_{n}, 0\right)=j\left(w_{n}\right), \quad W\left(0, e_{n}\right)=\left(F\left(w_{n}\right), w_{n}\right),
$$

then $W$ defines an isomorphism of $Z_{p}$ into $Z_{p}$.

Now consider the map $W-S V$. By construction, $(W-S V)\left(e_{n}, 0\right)=0$, $n>N$. Hence there is a subspace $Y$ of finite codimension such that ( $W-$ $S V) \mid Y$ factors through $q \mid Y$. It follows that $W-S V$ is strictly singular. As $W$ is an isomorphism, it follows that there exists an integer $n_{0}$ such that the restriction of $S V$ to $\overline{\operatorname{lin}}\left\{\left(e_{j}, 0\right),\left(0, e_{j}\right): j \geqslant n_{0}\right\}$ is an isomorphism. Hence $S$ is

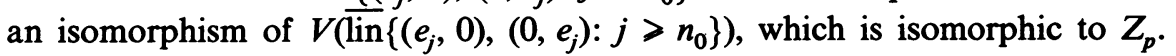
As remarked earlier, $T$ is then an isomorphism on a subspace isomorphic to $Z_{p}$, and the proof is complete.

COROllary 6.6. Every infinite-dimensional complemented subspace of $Z_{p}$ contains a subspace isomorphic to $Z_{p}$.

COROLLARY 6.7. $Z_{p}$ has no complemented subspace isomorphic to $l_{p}$.

CoROllaRY 6.8. $Z_{2}$ can not be embedded in any $L_{p}(1<p<\infty)$.

Proof. If $p>2$, then every Hilbertian subspace of $L_{p}$ is complemented in $L_{p}$ by a result of Kadec and Petczynski [3, 3. Corollary 1]; the result then follows from 6.7. Assume $1 \leqslant p \leqslant 2$ and $Z_{2}$ embeds in $L_{p}$; then by a theorem of Rosenthal [11, Theorem 8] $Z_{2}$ embeds in $L_{p^{\prime}}$ for some $p^{\prime}$ in $(1,2]$. Now by a theorem of PeIczynski and Rosenthal [6, Theorem 3.1], since $Z_{2}$ contains a Hilbertian subspace it contains a complemented Hilbertian subspace; again this violates Corollary 6.7. This finishes the proof. 
COROLlARY 6.9. $Z_{p}$ has no complemented infinite-dimensional subspace with an unconditional basis.

Proof. By 5.4, any basic sequence in $Z_{p}$ has a subsequence which spans either $l_{p}$ or $l_{f, p}$; if the original sequence is unconditional, the span of the subsequence is complemented in the span of the original sequence. Also, the space $l_{f, p}$ contains a complemented subspace isomorphic to $l_{p}$. (This follows from the fact that the function $x^{p}$ is in the set $E_{M, 1}$; see, for example, [5, p. 157].)

Corollary 6.7 and the above now give the desired conclusion.

REMARK. $Z_{p}$ has an unconditional decomposition into two-dimensional subspaces. To see this, simply take $E_{n}$ to be the span of the two vectors $\left(e_{n}, 0\right)$ and $\left(0, e_{n}\right), n=1,2, \ldots$; the spaces $E_{n}$ form the desired decomposition.

\section{REFERENCES}

1. T. Aoki, Locally bounded linear topological spaces, Proc. Imp. Acad. Tokyo 18 (1942), 588-594.

2. P. Enflo, J. Lindenstrauss, and G. Pisier, On the "three-space problem", Math. Scand. 36 (1975), 199-210.

2a. T. Figiel, J. Lindenstrauss, and V. Milman, The dimension of almost spherical sections of convex bodies, Acta Math. 139 (1977), 53-94.

3. M. I. Kadec and A. PeYcyzński, Bases, lacunary series and complemented subspaces in the spaces $L_{p}$, Studia Math. 21 (1962), 161-176.

4. N. J. Kalton, The three-space problem for locally bounded F-spaces, Compositio Math. 37 (1978), 243-276.

5. J. Lindenstrauss and L. Tzafriri, Classical Banach spaces, Vol. I, Ergebnisse der Mathematik und ihrer Grenzgebiete, Band 92, Springer-Verlag, Berlin, 1977.

6. A. PeYczyński and H. P. Rosenthal, Localization techniques in $L^{p}$ spaces, Studia Math. 52 (1975), 263-289.

7. M. Ribe, Examples for the nonlocally convex three space problem, Proc. Amer. Math. Soc. 73 (1979), 351-355.

8. J. W. Roberts, $A$ non locally convex $F$-space with the Hahn-Banach approximation property, Proc. Kent State Conference, Lecture Notes in Math., vol. 604, Springer-Verlag, Berlin and New York, 1977.

9. S. Rolewicz, On certain classes of linear metric spaces, Bull. Acad. Polon. Sci. Sér. Sci. Math. Astronom. Phys. 5 (1957), 471-473.

10. H. P. Rosenthal, On relatively disjoint families of measures, with some applications to Banach space theory, Studia Math. 37 (1970), 14-36.

11. _ On subspaces of $L^{p}$, Ann. of Math. 97 (1973), 344-373.

12. P. Turpin, Convexités dans les espaces vectoriels topologiques généraux, Dissertationes Math. 131 (1976).

Department of Mathematics, University College of Swansea, Singleton Park, SWANSEA, SA2-8PP, UNITED KINGDOM

Department of Mathematics, University of Illinois, Urbana, Illinois 61801 (Current address of N. T. Peck)

Current address (N. J. Kalton): Department of Mathematics, University of Missouri, Columbia, Missouri 65211 